Research Article

\title{
New Chaotic Oscillator Derived from Class C Single Transistor-Based Amplifier
}

\author{
Jiri Petrzela \\ Department of Radio Electronics, Faculty of Electrical Engineering and Communications, Brno University of Technology, \\ Brno 616 00, Czech Republic \\ Correspondence should be addressed to Jiri Petrzela; petrzelj@feec.vutbr.cz
}

Received 28 June 2020; Revised 20 October 2020; Accepted 31 October 2020; Published 11 November 2020

Academic Editor: Adel Ouannas

Copyright ( 2020 Jiri Petrzela. This is an open access article distributed under the Creative Commons Attribution License, which permits unrestricted use, distribution, and reproduction in any medium, provided the original work is properly cited.

This paper describes a new autonomous deterministic chaotic dynamical system having a single unstable saddle-spiral fixed point. A mathematical model originates in the fundamental structure of the class $\mathrm{C}$ amplifier. Evolution of robust strange attractors is conditioned by a bilateral nature of bipolar transistor with local polynomial or piecewise linear feedforward transconductance and high frequency of operation. Numerical analysis is supported by experimental verification and both results prove that chaos is neither a numerical artifact nor a long transient behaviour. Also, good accordance between theory and measurement has been observed.

\section{Introduction}

Heretofore, formation of the strange attractors was reported in many lumped electronic circuits, both isolated and driven. Chaotic waveforms have several fundamental properties: wideband and continuous frequency spectrum, sensitivity to small changes of initial conditions, dense state attractors with (unlike noise) non-integer metric dimension, and absence of closed-form analytic solution. Because of these features, especially presence of dominant harmonics in frequency spectrum, chaos can be observed in general models of all types of lumped analogue harmonic oscillators, regardless of type of active element used as energy source. For example, paper [1] describes a long-term unpredictable motion of the well-known Colpitts oscillator. Work [2] demonstrates existence of robust strange attractors unfolded during study of motion of the Hartley oscillator. Both oscillators require only single transistor and three accumulation elements for generation of the stable sinusoidal output voltages. Some interesting papers $[3,4]$ introduce a methodology on how to transform very popular Wien-bridge network configuration into chaotic oscillator. It turns out that simple mechanism dedicated to amplitude stabilization can cause both vector field stretching and folding required for the chaos evolution. In fact, the most famous chaotic electronic system, Chua's oscillator, can be considered as connection of LC parallel resonant tank, timing network, and nonlinear resistor. This nonlinear element can be either polynomial [5] or piecewise linear [6]. Both types of nonlinearities are practically implemented using several commercially available devices and are the only active components in designed chaotic circuits. Study [7] proves that pair of coupled accumulation elements can be substituted by a single higher-order element such as the frequency dependent negative resistor. Single third-order immittance element can be used to create linear part of the vector field as it is demonstrated in paper [8]. Systematic design procedure leading to robust chaotic oscillators with the passive only nonlinear elements is provided in a couple of cookbooks $[9,10]$. In these articles, nonlinear resistors have the ampere-voltage curve with single breakpoint and therefore serves as energy valve. Several papers utilize proposed approach to construct original structures of chaotic oscillators $[11,12]$.

Besides oscillators, unpredictable motion was detected in other naturally non-chaotic analogue building blocks. Recently, in the modern complex electronic systems, chance to observe robust chaos is strengthened because of high frequencies and 
amplitudes of useful processed signals. In such cases, parasitic properties (especially input and output impedances) of active elements necessarily introduce error terms in describing set of differential equations, lead to unexpected couplings between independent nodes, change values of working accumulation elements and increase mathematical order of circuit, cause additional functional nonlinearities or hysteresis effects on existing nonlinear transfer curves, etc. Let us provide a few examples of such systems. Presence of structurally stable strange attractors was confirmed in standard topologies of DCDC converters, namely, buck [13], cuk [14], and boost [15, 16]. Very interesting reading about irregular behaviour in general class of DC-DC converters and its properties, including possible control mechanism, can be found in papers $[17,18]$. Another transparent example of chaotic system based on switched power converter is described in study [19]. Impulsive chaos can be observed in switched capacitor circuits as well; see papers $[20,21]$ for details. Several research papers were dedicated to describing chaos in phase-locked loops: for details, consult several examples provided in papers [22, 23]. Recently, single-spiral as well as double-scroll attractors were detected within high frequency mathematical models of multistate static memory cells composed of the resonant tunnelling diodes; the number of these diodes determines total amount of fixed points. Chaos can be observed if ampere-voltage characteristic is considered piecewise linear [24] as well as cubic polynomial [25]. An interesting consequence coming from these studies is that there is a direct correlation between number of equilibria and complexity of generated chaotic attractors. Very complex vector field and geometric patterns of basins of attraction [26] suggest possible evolution of multiscroll chaotic attractors. Fingerprints of chaos can be observed in very simple networks that contain recently developed memory circuit elements: memristors $[27,28]$ or mem-elements in general $[29,30]$, both with integer and fractional order [31]. To end this, very brief, list of chaotic analogue building blocks, we can mention frequency filters as good candidates to exhibit chaotic solution. These filtering sections can be of different topologies, such as the state variable filters [32] or passive ladder filters with active nonlinear feedback. This active nonlinear feedback can be implemented by either two-terminal $[5,33,34]$ or two-port [35] device. There, existence of chaos is conditioned by small system dissipation, i.e., filters with high quality factor. Speaking in terms of lumped chaotic circuit with minimum number of elements, it seems that driven R-L-diode circuit is the simplest one [36].

Searching for the most trivial algebraic description of continuous time chaotic flow was started by fundamental question raised in [37]. To date, this problem still represents up-to-date topic.

Existence of robust chaotic waveforms within very simple lumped electronic systems having one or two transistors is old but still an up-to-date problem. Some recent examples of transistor-based chaotic oscillators can be found in paper [38]. Circuits discussed here clearly belong to the simplest cases exhibiting chaotic dynamics because, apart from the transistor used, some comprise only three accumulation elements. A slightly more complicated chaotic network having two standard bipolar transistors is discussed in research work [39].
The main purpose of upcoming sections of this paper is to prove that chaos can be observed, under very specific conditions, in the fundamental cell of class $\mathrm{C}$ amplifier comprising single bipolar transistor. This fact has been already mentioned in a very recent publication [40]. Unlike this research, where both backward and forward transconductances of bipolar transistor two-port are considered as polynomial nonlinearity, this work assumes a case much closer to physical reality. In addition, two differently shaped strange attractors instead of one are presented and thoroughly analysed. Although describing mathematical model that gives basis to this work and corresponding circuit realization is not the simplest one, it still represents interesting chaotic dynamical system that belongs to class of systems having a single unstable equilibrium.

This paper is organized as follows. The next section provides a brief mathematical description of autonomous deterministic dynamical system to be analysed. The third section of this paper numerically quantifies chaotic motion produced by analysed system. The fourth section describes two flow-equivalent circuit designs based on class $\mathrm{C}$ amplifier cell with polynomial nonlinearity. The fifth section of this manuscript is dedicated to experimental verification of constructed chaotic oscillators. Finally, concluding remarks and future possible topics are revealed.

\section{Mathematical Background}

The principal structure of class $\mathrm{C}$ single transistor-based amplifier is given in Figure 1(a). For useful signals, this network can be redrawn as computational model shown in Figure 1(b). In its basic form, both circuits are linear and valid for small useful signals. Without input waveform, the resulting dynamical system can be described by the following set of ordinary differential equations:

$$
\begin{aligned}
C_{\mathrm{be}} \frac{\mathrm{d}}{\mathrm{d} t} v_{1} & =-y_{11} \cdot v_{1}-y_{12} \cdot v_{2}, \\
\left(C+C_{\mathrm{ce}}\right) \frac{\mathrm{d}}{\mathrm{d} t} v_{2} & =-y_{21}\left(v_{1}\right)-y_{22} \cdot v_{2}-i_{L}, \\
L \frac{\mathrm{d}}{\mathrm{d} t} i_{L} & =v_{2} .
\end{aligned}
$$

Note that admittance parameters of bipolar transistor $y_{11}, y_{12}$, and $y_{22}$ are considered constant. This represents common situation in many practical applications. Forward transconductance is supposed to be nonlinear, characterized by curve approximated by cubic polynomial of form $y_{21}\left(v_{1}\right)=a \cdot v_{1}^{3}+b \cdot v_{1}$. Symbolic form of Jacobi matrix is

$$
\mathbf{J}=\left(\begin{array}{ccc}
-\frac{y_{11}}{C_{\mathrm{be}}} & -\frac{y_{12}}{C_{\mathrm{be}}} & 0 \\
-\frac{b}{\left(C+C_{\mathrm{ce}}\right)}-\frac{y_{22}}{\left(C+C_{\mathrm{ce}}\right)}-\frac{1}{\left(C+C_{\mathrm{ce}}\right)} \\
\frac{1}{L} & 0
\end{array}\right),
$$




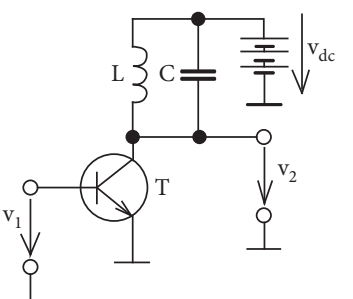

(a)

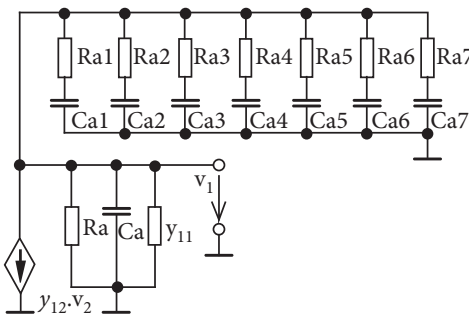

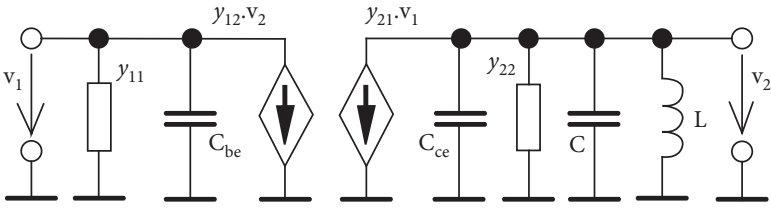

(b)

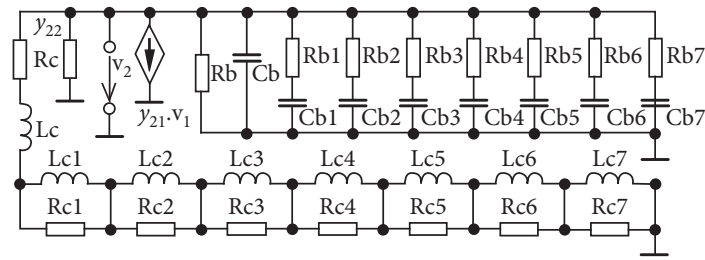

(c)

FIGURE 1: Class C amplifier with single bipolar transistor: (a) conventional simplifier connection, (b) concept considering transistor as twoport described by scalar linear admittance parameters, (c) equivalent circuit for non-integer-order accumulation elements in class $\mathrm{C}$ amplifier.

and characteristic polynomial calculated for single fixed point located at origin is

$$
\begin{aligned}
\operatorname{det}(\lambda \cdot \mathbf{E}-\mathbf{J})= & \lambda^{3}+\left(\frac{y_{11}}{C_{\mathrm{be}}}+\frac{y_{22}}{C+C_{\mathrm{ce}}}\right) \lambda^{2} \\
& +\left(\frac{1}{\left(C+C_{\mathrm{ce}}\right) L}+\frac{y_{11} \cdot y_{22}-b \cdot y_{12}}{C_{\mathrm{be}}\left(C+C_{\mathrm{ce}}\right)}\right) \lambda \\
& +\frac{y_{11}}{C_{\mathrm{be}}\left(C+C_{\mathrm{ce}}\right) L}=0 .
\end{aligned}
$$

In further text, let us assume that biasing point forces transistor to work in a regime of constant current source; i.e., $y_{22}=0 \mathrm{~S}$. Also, suppose that normalized values of accumulation elements are $C_{\mathrm{ce}}=0 \mathrm{~F}, C_{\mathrm{be}}=C=1 \mathrm{~F}, L=1 \mathrm{H}$ and backward transconductance is linear; i.e., $y_{12}=1 \mathrm{~S}$. Considering this, characteristic polynomial simplifies into

$$
\gamma: \lambda^{3}+y_{11} \cdot \lambda^{2}+(1-b) \lambda+y_{11}=0 .
$$

For the purpose of the upcoming optimization, namely, to reduce size of hyperspace of parameters that undergoes search, limit cases for sought parameters become important

$$
\begin{gathered}
\lim _{y_{11} \longrightarrow 0} \operatorname{roots}(\gamma): \lambda_{1,2}=\mp \sqrt{b-1}, \quad \lambda_{3}=0, \\
\lim _{b \longrightarrow 0} \operatorname{roots}(\gamma): \lambda_{1,2}=\mp j, \quad \lambda_{3}=y_{11} .
\end{gathered}
$$

\section{Numerical Analysis}

All numerical integration results presented in this paper were achieved by using four-order Runge-Kutta method with fixed step size. This routine was also used as core for calculation of the largest Lyapunov exponent (LLE) [41] together with Gram-Smith orthogonalization. LLE as the flow quantifier forms one fitness function for non-gradient optimization as admitted in papers [42, 43]. In fact, searching for chaotic solution of dynamical system is a multi-objective optimization problem. Firstly, chaotic behaviour should not be interchanged with long transient motion. Therefore, LLE needs to be calculated on the attractor. Also, slowly divergent motion needs to be removed from interesting solution. These potentially useful results are stored and finally verified by handmade analysis and attractor visualization. Boundedness of numerically integrated $\omega$-limit set is verified via inside-sphere condition. Whole optimization routine was implemented in MATLAB. Since evaluation of individual objective functions is not mutually connected, searching process could be significantly accelerated by employing CUDA-based parallel processing. Nevertheless, searching within three-dimensional space of internal system parameters is still a very demanding operation.

Note that the optimization procedure described above and its results, i.e., numerical values of input admittance of transistor and shape of the cubic polynomial forward transconductance, do not have direct correlation with physical nature of one stage class $\mathrm{C}$ amplifier. However, admittance is a scalable network quantity (via impedance norm) and, for the large processed amplitudes of useful signal, saturation-type amplification property of bipolar transistor is mild hypothesis. Of course, normalized value of $y_{12}=1 \mathrm{~S}$ indicates strongly non-unilateral bipolar transistor stage.

Two sets of internal parameters leading to different shapes of strange attractors and associated non-integer 
Kaplan-Yorke dimension $D_{K Y}$ have been discovered, namely,

$$
\begin{aligned}
y_{11} & =0.2, \\
a & =-0.8, \\
b & =2.56, \\
\mathrm{LE}_{\max } & =0.061, \\
D_{\mathrm{KY}} & =2.234, \\
y_{11} & =0.2, \\
a & =-0.8, \\
b & =3.5, \\
\mathrm{LE}_{\max } & =0.072, \\
D_{\mathrm{KY}} & =2.127 .
\end{aligned}
$$

Eigenvalues associated with fixed point for dynamical system with (6) are

$$
\begin{aligned}
& \lambda_{1}=-1.408, \\
& \lambda_{2}=1.077, \\
& \lambda_{3}=0.132,
\end{aligned}
$$

and for set (7) we get

$$
\begin{aligned}
& \lambda_{1}=-1.721, \\
& \lambda_{2}=1.44, \\
& \lambda_{3}=0.081 .
\end{aligned}
$$

Therefore, origin is saddle-node equilibrium point with stability index 1.

Figure 2 demonstrates shapes of typical strange attractors generated by dynamical system (1) with parameters (6). Numerical integration was calculated with final time $300 \mathrm{~s}$ and time step $10 \mathrm{~ms}$. Because of vector field symmetry with respect to the horizontal plane $z=0$, there are two mirrored strange attractors with the same geometric structure. Figure 2 also shows sensitivity to the small deviations in the initial conditions and vector field near fixed point $\mathbf{x}_{\mathrm{e}}=(0,0$, $0)^{T}$. For this analysis, final time and time step were kept the same. Figure 3 is a visualization of LLE as two-dimensional function of shape of forward transconductance function $y_{21}\left(v_{1}\right)$. For values of input admittance $y_{11}$ bigger than 0.6, chaos disappears, and class $\mathrm{C}$ amplifier produces various limit cycles. Order in circuit is restored thanks to the significant system dissipation. Of course, cases with $y_{11} \leq 0$ are out of the question because of the physical essence of the input admittance. Note that hidden attractors including those with fractal geometric dimension are not excluded by investigations presented in this paper. Figure 4 shows distribution of dynamic energy over state space volume occupied by strange attractor. To visualize the local dynamics calculated at each point in $510 \times 610$ grid, numerical integration was up to final time $1 \mathrm{~s}$ with time step $10 \mathrm{~ms}$. Value ranges for individual edges are uniform: $v_{1} \in(-2.5,2.5)$ and $v_{2} \in(-3,3)$.
Figure 5 provides information about shape of the strange attractor if dynamical system (1) has the internal parameters (7). It strongly resembles the so-called double hook attractor that can be generated by Chua's circuit with three real eigenvalues associated with fixed point located at origin [44]. Unlike Chua's circuit, there are two coexisting double hooks. Also note evolution of two neighbouring orbits plotted with respect to time: difference is visible after quite a long time; about $120 \mathrm{~s}$ lapses to phase shift and $170 \mathrm{~s}$ expires to observe exponential divergence.

3.1. Fractional-Order Case. Recently, discovery and presentation of new chaotic dynamical system are usually supported by numerical analysis of describing mathematical model under condition of fractionality; that is, one or all differential equations are of non-integer order. Such investigation could be performed on three levels. The first level is pure mathematical and involves discretization of ordinary [45] or partial [46] differential equations and performing iterations. This numerical approach is well suited for chaotic [47-49] as well as hyperchaotic [50,51] dynamics. The second level is to model fractional-order (FO) circuit element in the form of two-terminal or twoport using suitable circuit-oriented software such as OrCAD PSpice or Multisim. Both classes of FO elements (often called constant phase elements due to their fundamental properties keeping constant phase shift between response and driving force) could be considered either ideal or approximated in the frequency domain. To reach precise approximation of FO two-terminal fully passive circuit element, paper [52] could be consulted. Design procedure toward approximated FO two-port element is provided in a comprehensive study [53]. Since chaotic signal is wideband, the mentioned approximations should be valid, at least, within the same frequency range. To avoid laborious calculations, passive ladder FO capacitors of different math orders can be found in [54].

Because of practically oriented concluding remarks coming from this research study, the last method dedicated for modelling non-integer nature of accumulation elements associated with class $\mathrm{C}$ amplifier has been adopted. New circuit structure to be analysed is provided in Figure 1(c). Note that FO nature of accumulation elements is modelled by fully passive ladder networks having seven sections. This number turns to be enough, from the viewpoint of both required frequency range (five decades) and maximal allowed phase error (that is lower than 1 degree). To increase universality, let us denote mathematical order of the approximation associated with capacitor $C_{\mathrm{be}}$ by $k$, sum of capacitors $C+C_{\mathrm{ce}}$ by letter $m$, and math order of inductor by $n$. State vector changes into a more complex form

$$
x=\left(v_{1}, v_{a 1}, \ldots, v_{a k}, v_{2}, v_{b 1}, \ldots, v_{b m}, i_{L}, i_{c 1}, \ldots, i_{c n}\right)^{T} .
$$




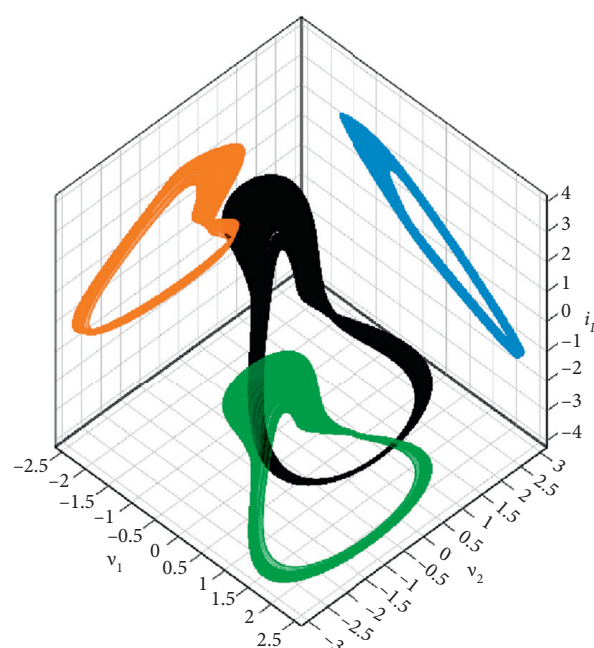

(a)

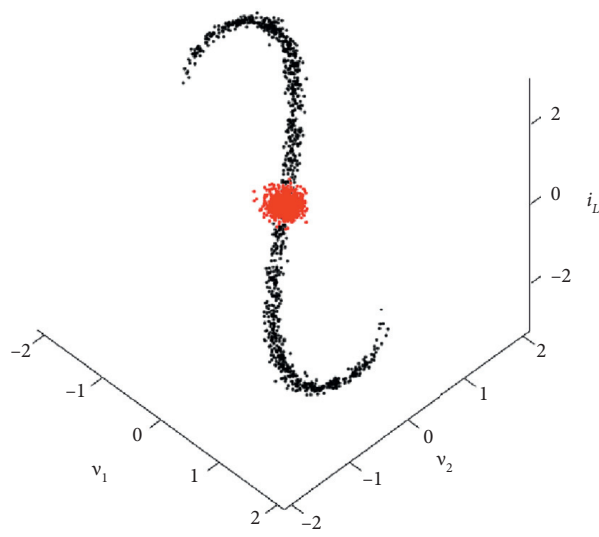

(c)

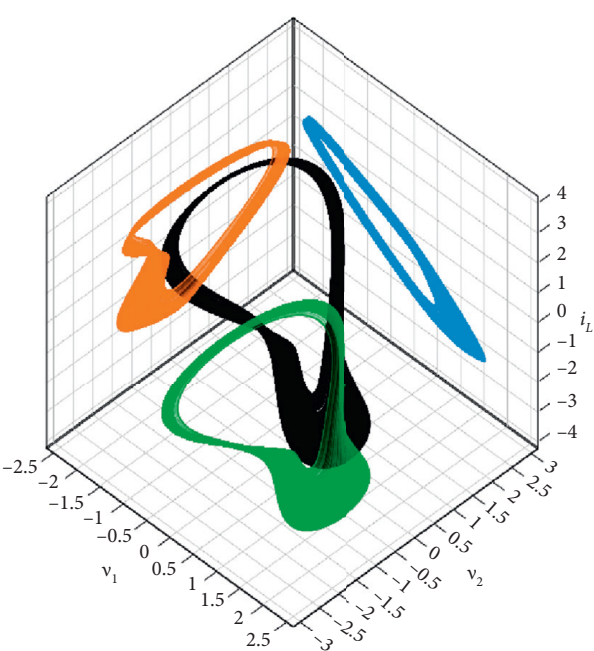

(b)
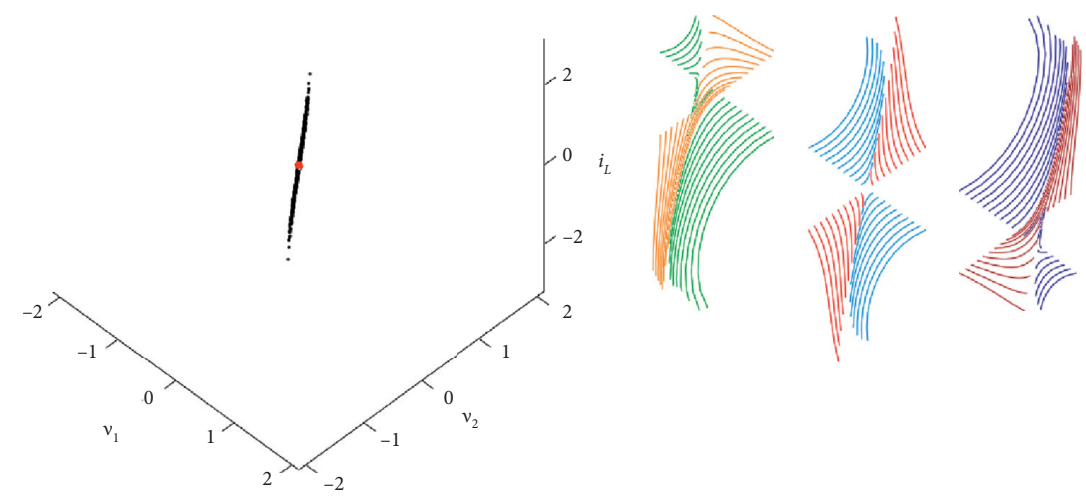

(d) (e)

(f) (g)

FIGURE 2: Numerical integration leading to typical chaotic attractors generated by fundamental class C amplifier: $(\mathrm{a}) x_{0}=(0,0.5,0)^{T}$, and $(\mathrm{b})$ $x_{0}=(0,-0.5,0)^{T}$. Final states (black dots) that represent sensitivity to changes of initial conditions (red dots), normal distribution of the initial states around $x_{0}=(0,0.5,0)^{T}$ with the standard deviation: (c) $\sigma=0.01$, and (d) $\sigma=0.1$. Local behaviour close to fixed point: (e) plane $z_{0}=1$, line $y_{0}=0$ (green) and line $x_{0}=0$ (orange), (f) plane $z_{0}=0$, line $y_{0}=0$ (blue), line $x_{0}=0$ (red), and (g) plane $z_{0}=-1$, line $x_{0}=0$ (dark blue), line $x_{0}=0$ (brown).

Behaviour of this lumped electronic system is uniquely determined by the following set of ordinary differential equations:

$$
\begin{gathered}
\frac{\mathrm{d}}{\mathrm{d} t} v_{1}=-\frac{1}{C_{a}}\left(\frac{1}{R_{a}}+y_{11}\right) v_{1}-\frac{y_{12}}{C_{a}} v_{2}+\frac{1}{C_{a}} \sum_{j=1}^{k} \frac{1}{R_{a j}}\left(v_{a j}-v_{1}\right), \\
x=1,2, \ldots, k: \frac{\mathrm{d}}{\mathrm{d} t} v_{a x}=\frac{1}{C_{a x} R_{a x}}\left(v_{1}-v_{a x}\right), \\
\frac{\mathrm{d}}{\mathrm{d} t} v_{2}=-\frac{1}{C_{b}}\left(\frac{1}{R_{b}}+y_{22}\right) v_{2}-\frac{y_{21}\left(v_{1}\right)}{C_{b}}+\frac{1}{C_{b}} \sum_{j=1}^{m} \frac{1}{R_{a j}}\left(v_{b j}-v_{2}\right),
\end{gathered}
$$

$$
x=1,2, \ldots, m: \frac{\mathrm{d}}{\mathrm{d} t} v_{a x}=\frac{1}{C_{a x} R_{a x}}\left(v_{2}-v_{a x}\right),
$$

where $v_{a j}$ and $v_{b j}$ stand for voltage across $j^{\text {th }}$ capacitor in approximation of $C_{\mathrm{be}}$ and $C+C_{\mathrm{ce}} \mathrm{FO}$ capacitor, respectively. Similarly, symbol $i_{c j}$ represents current flowing through $j^{\text {th }}$ inductor. Note that second, fourth, and sixth differential equation are presented in our system $k^{\text {th }}, m^{\text {th }}$, and $n^{\text {th }}$ times, respectively. Obviously, we are experiencing system of $3+k+m+n$ first-order ordinary differential equations. Thus, instead of calculation of the binomial coefficients and discretization of differential equations, we can follow the standard way of numerical integration process. Some numerical results are graphically demonstrated via Figure 6. For mathematical orders of the approximated constant 


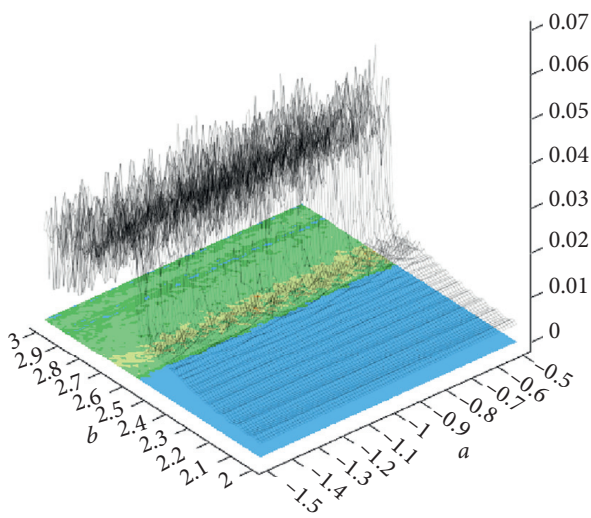

(a)

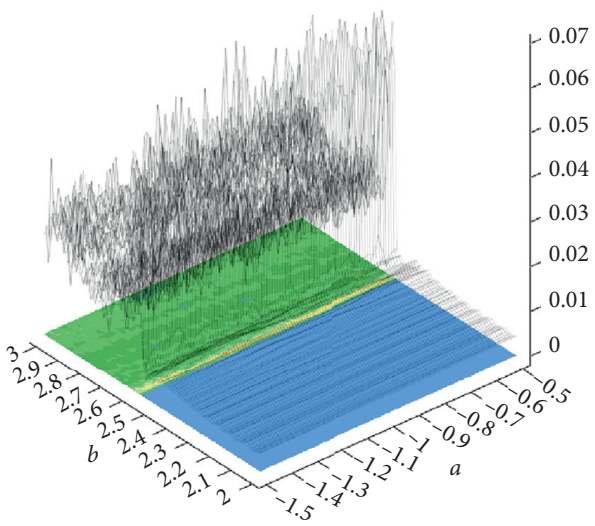

(c)

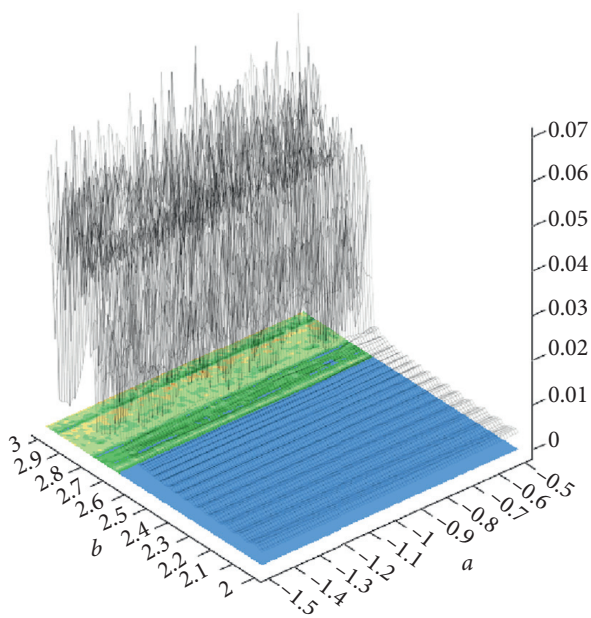

(e)

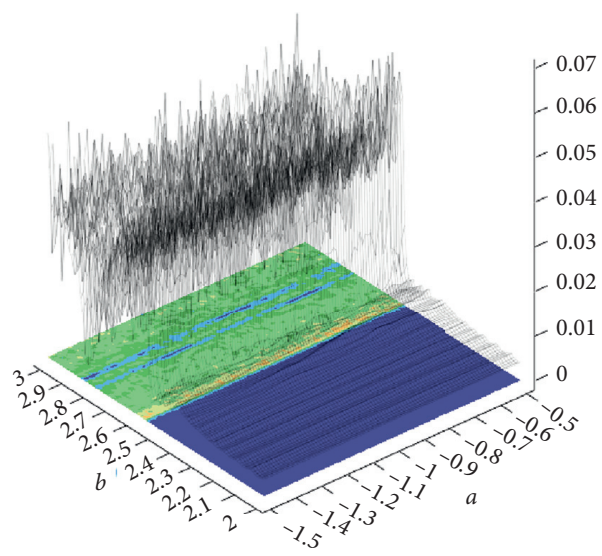

(b)

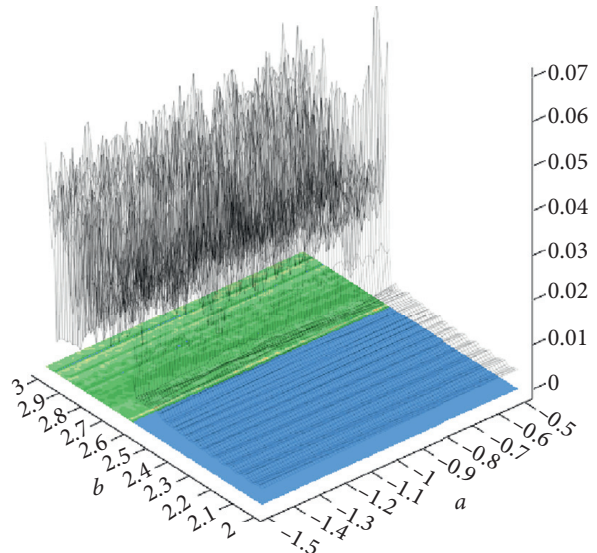

(d)

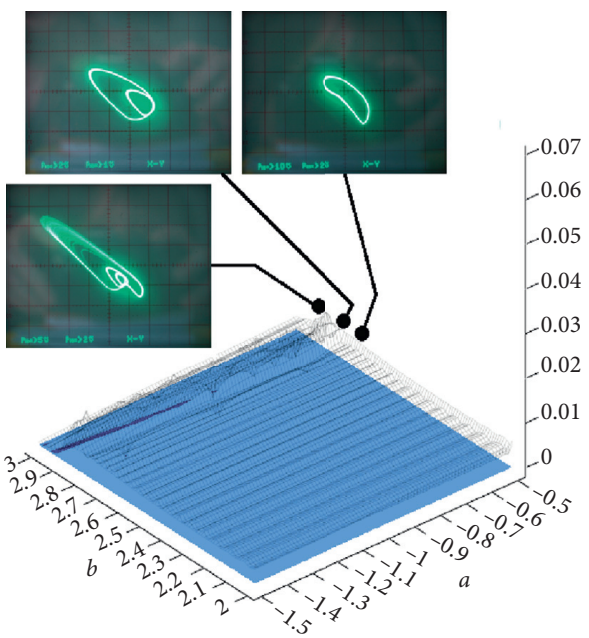

(f)

FIgURE 3: Topographically scaled surface-contour plot of LLE as a function of polynomial coefficients for different values of system dissipation: (a) $y_{11}=0.1$, (b) $y_{11}=0.2$, (c) $y_{11}=0.3$, (d) $y_{11}=0.4$, (e) $y_{11}=0.5$, and (f) $y_{11}=0.6$. Oscilloscope screenshots provided here demonstrate route-to-chaos scenario via period doubling sequence associated with class $\mathrm{C}$ amplifier having the largest system dissipation considered in this manuscript.

phase elements below 0.9 , chaotic behaviour of class $\mathrm{C}$ amplifier disappears and for very low total orders of circuit only limit cycles were observed. Symbols $\alpha, \beta$, and $\delta$ used in Figure 6 represent FO of first, second, and third differential equation, respectively. Let us discuss a specific FO case where orders of individual differential equations equal 0.9.

By adopting numerical values provided in paper [54] for mathematical order 0.9 , frequency range of constant phase 


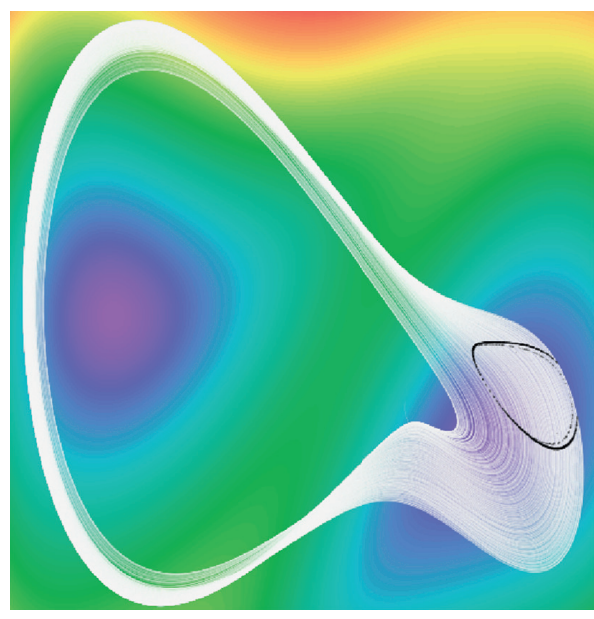

(a)

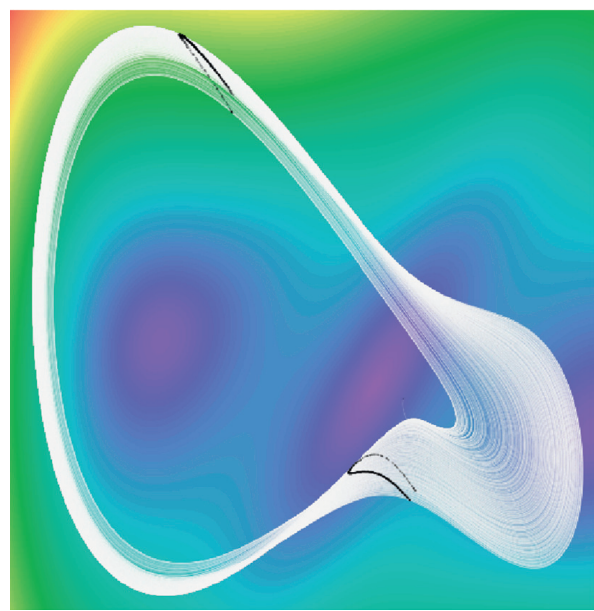

(c)

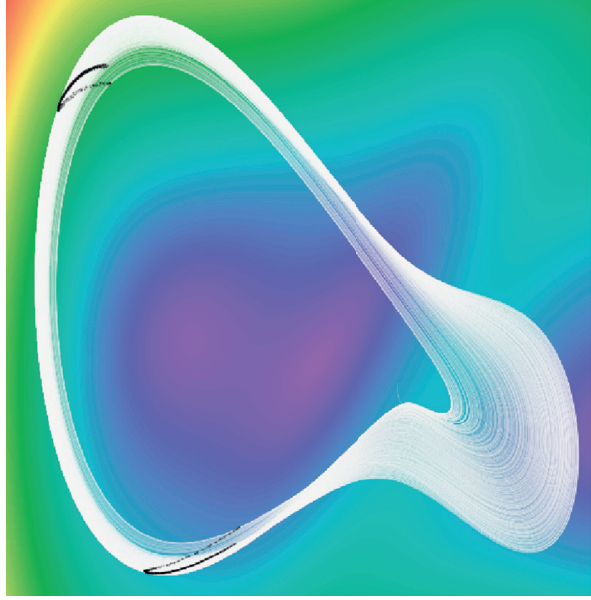

(e)

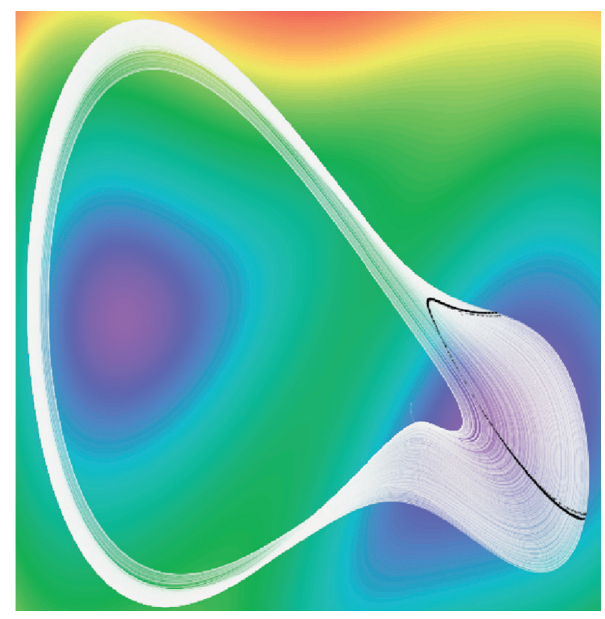

(b)

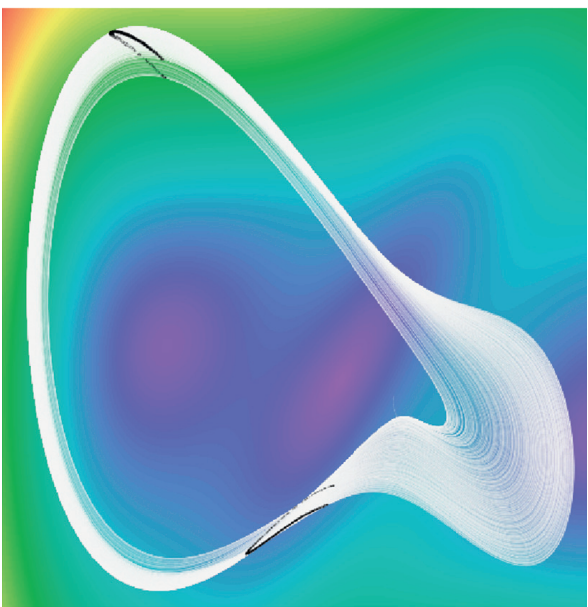

(d)

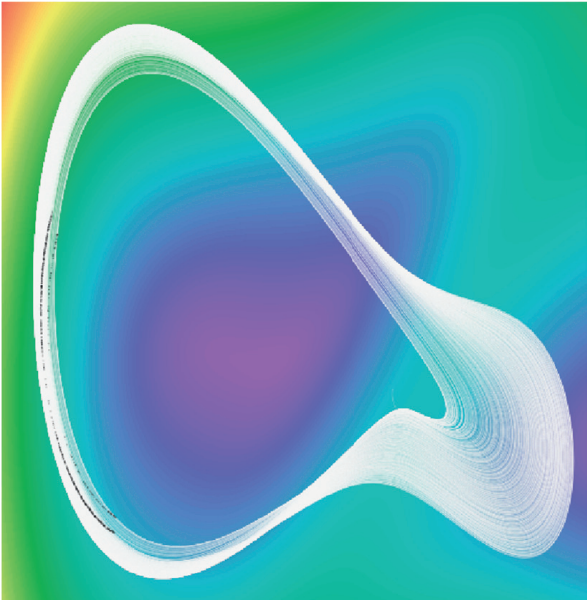

(f)

FIGURE 4: Rainbow scaled plots showing distribution of the kinetic energy over the state space, horizontal slices: (a) $z_{0}=-2.3$, (b) $z_{0}=-2$, (c) $z_{0}=0$, (d) $z_{0}=0.5$, (e) $z_{0}=1$, and (f) $z_{0}=1.5$. Colour scale is as follows: very high energy (red), high energy (yellow), average energy (green), low energy (blue), very low energy (purple). Black dots represent return maps for the Poincaré sections equivalent to horizontal slices mentioned above. 
element can be downscaled from original interval $10 \mathrm{~Hz}$ up to $1 \mathrm{MHz}$. To analyse normalized circuit, pseudo-capacitance of the constant phase element will be $1 \mathrm{~s}^{0.1} / \mathrm{F}$ (FO capacitor) and pseudo-inductance approximately equals $1 \mathrm{~s}^{0.1} / \mathrm{F}$ (FO inductor). For the first case, resulting numerical values of resistors and capacitors will be

$$
\begin{aligned}
R_{a} & =1.19 \Omega, \\
R_{a 1} & =0.21 \Omega, \\
R_{a 2} & =0.031 \Omega, \\
R_{a 3} & =4.579 \cdot 10^{-3} \Omega, \\
R_{a 4} & =6.79 \cdot 10^{-4} \Omega, \\
R_{a 5} & =1.008 \cdot 10^{-4} \Omega, \\
R_{a 6} & =1.498 \cdot 10^{-5} \Omega, \\
R_{a 7} & =2.24 \cdot 10^{-6} \Omega, \\
C_{a} & =0.206 \mathrm{~F}, \\
C_{a 1} & =0.171 \mathrm{~F}, \\
C_{a 2} & =0.14 \mathrm{~F}, \\
C_{a 3} & =0.113 \mathrm{~F}, \\
C_{a 4} & =0.091 \mathrm{~F}, \\
C_{a 5} & =0.074 \mathrm{~F}, \\
C_{a 6} & =0.06 \mathrm{~F}, \\
C_{a 7} & =0.053 \mathrm{~F} .
\end{aligned}
$$

Of course, these values hold for second FO capacitor as well; i.e., index $a$ can be replaced by $b$. FO inductor can be modelled by values

$$
\begin{aligned}
R_{c} & =2.4 \Omega, \\
R_{a 1} & =12 \Omega, \\
R_{a 2} & =63 \Omega, \\
R_{a 3} & =333.6 \Omega, \\
R_{a 4} & =1716 \Omega, \\
R_{a 5} & =8850 \Omega, \\
R_{a 6} & =4.62 \cdot 10^{4} \Omega, \\
R_{a 7} & =8.4 \cdot 10^{4} \Omega, \\
L_{c} & =0.198 \mathrm{H}, \\
L_{c 1} & =0.12 \mathrm{H}, \\
L_{c 2} & =0.102 \mathrm{H}, \\
L_{c 3} & =0.084 \mathrm{H}, \\
L_{c 4} & =0.072 \mathrm{H}, \\
L_{c 5} & =0.058 \mathrm{H}, \\
L_{c 6} & =0.048 \mathrm{H}, \\
L_{c 7} & =0.04 \mathrm{H} .
\end{aligned}
$$

LLE can be utilized to obtain piecewise linear approximation of a smooth polynomial vector field [55]. In our case, transconductance $y_{21}(v)$ could be substituted by the three-segment odd-symmetrical function with parameters

$$
\begin{aligned}
&\left|v_{1}\right| \leq V_{\mathrm{BP}}: y_{21}\left(v_{1}\right)=g_{\text {in }} \cdot v_{1}, \\
& v_{1}>V_{\mathrm{BP}}: y_{21}\left(v_{1}\right)=g_{\text {out }}\left(v_{1}-V_{\mathrm{BP}}\right)+g_{\text {in }} \cdot V_{\mathrm{BP}}, \\
& v_{1}<-V_{\mathrm{BP}}: y_{21}\left(v_{1}\right)=g_{\text {out }}\left(v_{1}+V_{\mathrm{BP}}\right)-g_{\text {in }} \cdot V_{\mathrm{BP}} .
\end{aligned}
$$

For the first discovered set of "chaotic" parameters (6), piecewise linear approximation can be adopted with

$$
\begin{gathered}
V_{\mathrm{BP}}=1.2, \\
g_{\text {in }}=1.9, \\
g_{\text {out }}=-6,
\end{gathered}
$$

while for the second set of parameters (7) leading to evolution of robust chaos optimal piecewise linear approximation is

$$
\begin{gathered}
V_{\mathrm{BP}}=1.3, \\
g_{\text {in }}=2.2, \\
g_{\text {out }}=-7 .
\end{gathered}
$$

The corresponding strange attractor is provided in Figure 7 together with the plot of dynamic energy distribution over three horizontal planes of state space. Note the high degree of attractor similarity with the results provided in Figure 5.

An interesting question that could be raised here is the possibility to generate the so-called multi-scroll [56] or multi-grid [57] strange attractors. This kind of complex behaviour is associated with specific geometry of vector field. State space can be divided into "periodic" formation of the affine segments with the same properties: existence of single fixed point with saddle-spiral local geometry with stability index 1. In each mentioned segment, state trajectory is attracted toward eigen-plane and spirals away to another segment. Famous double-scroll attractor observed in Chua's oscillator can be considered as ancestor for this kind of motion and special type of multi-spiral attractor. However, eigenvalues associated with parameter sets (15) are

$$
\begin{aligned}
& \lambda_{1}=-1.142, \\
& \lambda_{2}=0.687, \\
& \lambda_{3}=0.255,
\end{aligned}
$$

and for set (16) we obtain

$$
\begin{aligned}
& \lambda_{1}=-1.269, \\
& \lambda_{2}=0.893, \\
& \lambda_{3}=0.176 .
\end{aligned}
$$

These eigenvalues do not lead to vector field configuration promising from the viewpoint of generation of multi-spiral attractor. Also, numerical investigation does not reveal such kind of dynamical motion. In outer regions of the state space, i.e., if $v_{1}<-V_{\mathrm{BP}}$ or $v_{1}>V_{\mathrm{BP}}$, dynamical system exhibits strong dissipation along all directions and state orbit is pushed toward inner segment as demonstrated by means of Figure 8 . 


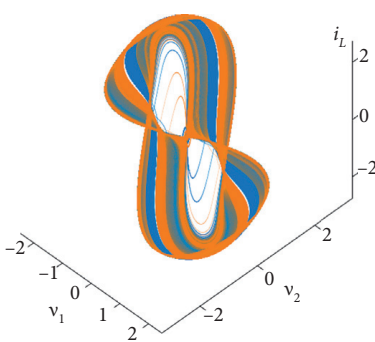

(a)

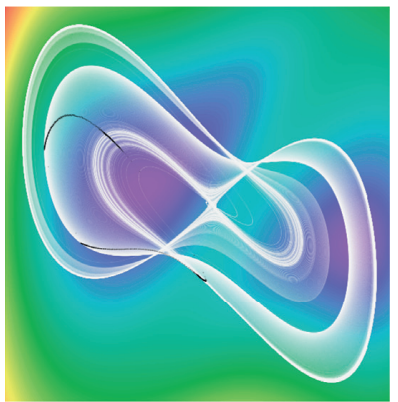

(e)

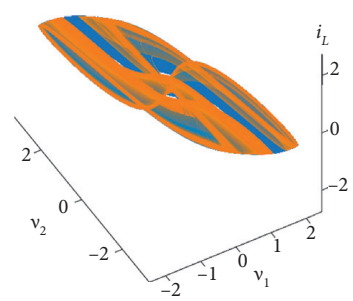

(b)

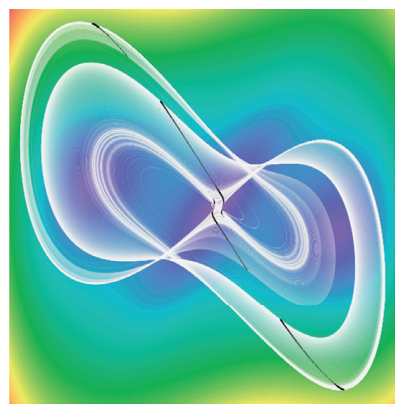

(c)

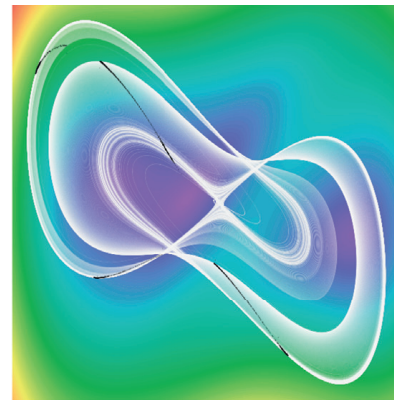

(d)

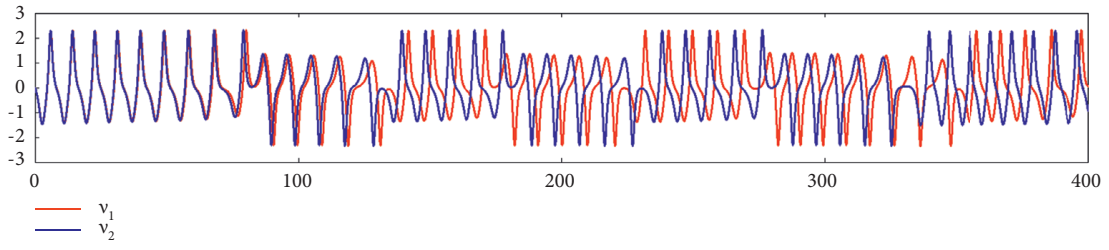

(f)

FIGURE 5: Brief numerical analysis of chaotic system (1) considering second discovered set of parameters (7): (a) (b) perspective visualization of generated strange attractor. Rainbow scaled distribution of kinetic energy measured in plane: (c) $z=0$, (d) $z=1$, and (e) $z=2$. Black dots are return maps plotted at the corresponding planes. Generated waveforms in time domain (f): red curve for $x_{0}=(0,0.5,0)^{T}$ and blue curve for $x_{0}=(0,0.51,0)^{T}$.

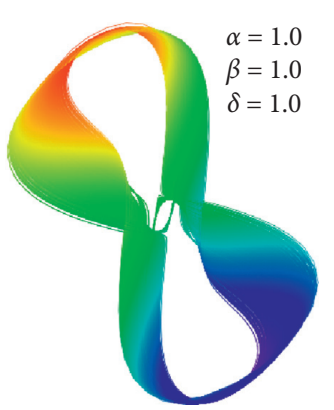

(a)

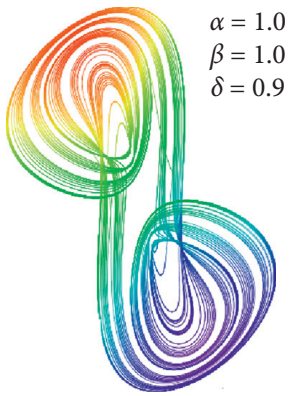

(b)

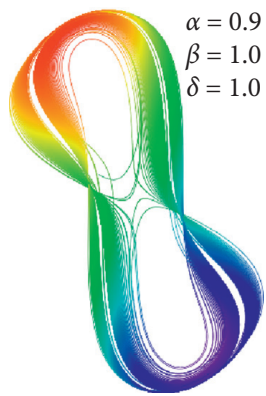

(c)

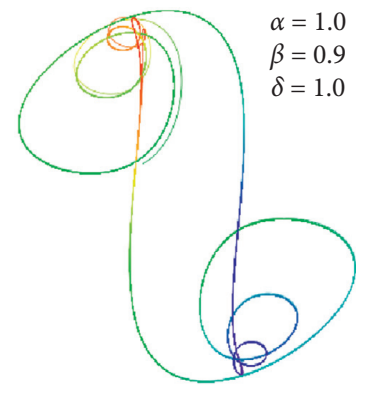

(d)

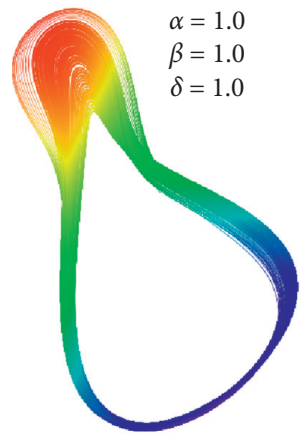

(e)

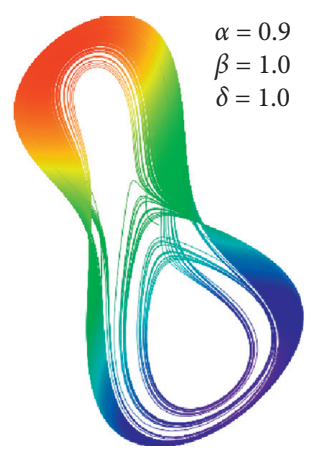

(f)

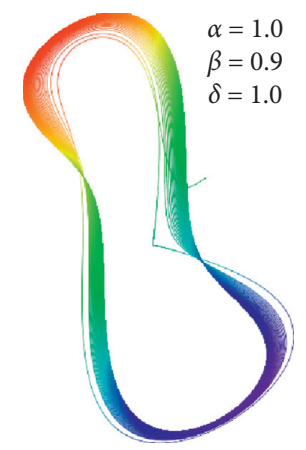

(g)

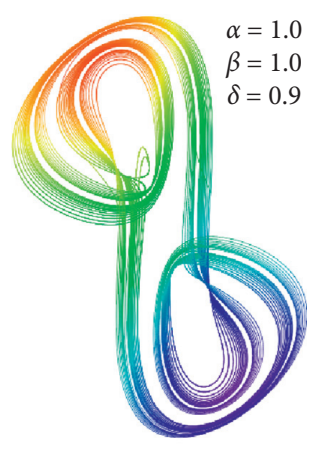

(h)

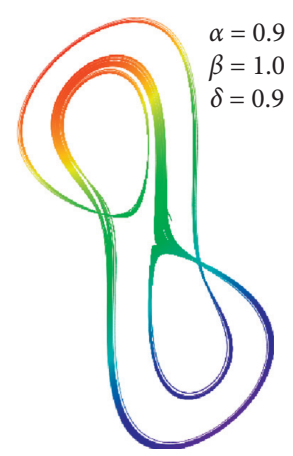

(i)

FIgure 6: Numerical investigation of class C amplifier with constant phase elements, selected Mathcad-based results: for (a), (b), (c), (d), parameter set (6) was adopted, and plots (e) (f), (g), (h), (i) are bounded to parameter group (7). 


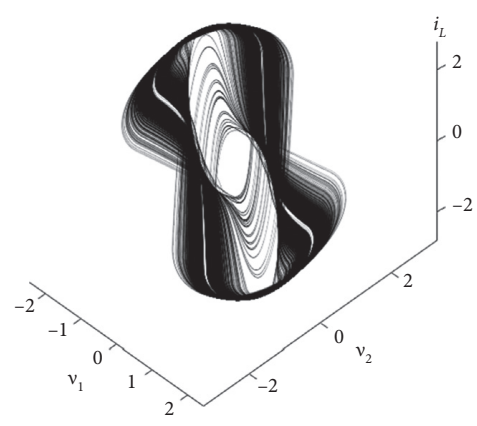

(a)

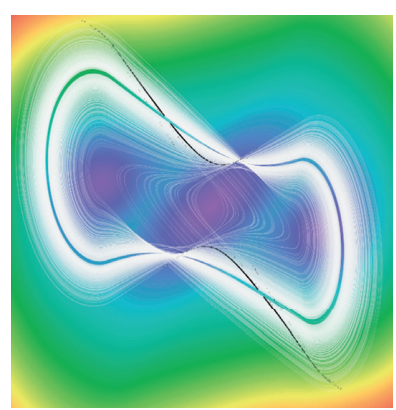

(b)

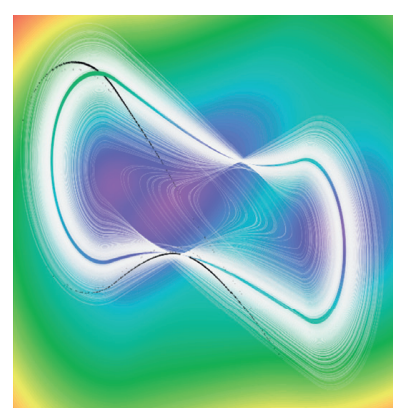

(c)

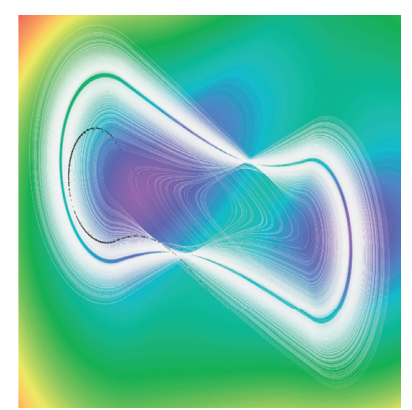

(d)

Figure 7: Brief numerical analysis of the piecewise linear case of the chaotic system: (a) perspective visualization of generated strange attractor. Rainbow scaled distribution of kinetic energy measured in plane: (b) $z=0$, (c) $z=1$, and (d) $z=2$. Black dots are return maps plotted at the corresponding planes.

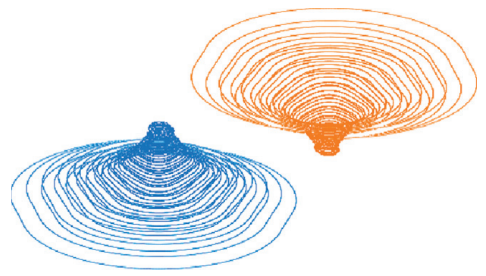

(a)

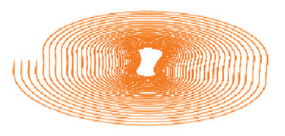

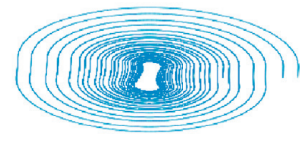

(b)

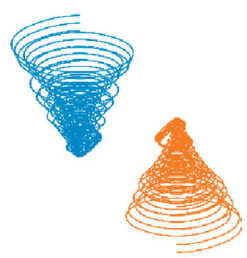

(c)

FIGURE 8: Convergency of state trajectory toward inner segment of vector field with positive (orange) and negative (blue) displacement of initial conditions in direction of (a) voltage $v_{1}$, (b) voltage $v_{2}$, and (c) current $i_{L}$.

Figure 9 demonstrates complex nature of basins of attraction calculated for dynamical system (1) with parameter group (6). Individual colours distinguish between three $\omega$-limit sets: chaotic attractor (red), solution close to limit cycle (green), and fixed point (black). Due to vector field symmetry, only horizontal state space slices having $z \geq 0$ are provided. Figure 10 represents the same plots but related to parameter set (7).

By introducing constant term to polynomial function $y_{21}\left(v_{1}\right)=a \cdot v_{1}^{3}+b \cdot v_{1}+c$, fixed point located at the origin turns into virtual. For small values of parameter $c$, this equilibrium with less dynamical system still generates strange attractors like the original. Thus, there is the overlap of discovered chaotic dynamical system into quite rare cases without equilibrium.

\section{Circuit Design}

To meet the basic criteria for presentation of new chaotic system [58], structural stability of desired strange attractor needs to be proven. Because of limited capability of human eye to catch short time transient movement, practical construction and laboratory verification of chaotic oscillator is the best way how to demonstrate such stability.

If a set of ordinary differential equations and numerical values of internal parameters is known, the problem of circuit synthesis belongs to simple and straightforward task with multiple correct solutions. If minimization of circuits elements is not design priority, we can follow the concept of analogue computers. Only three building blocks are required: inverting integrators, differential amplifiers, and two-ports with a nonlinear transfer characteristic. Final network can work in either voltage $[59,60]$ or current mode [61]. Under specific circumstances, resulting circuit can be simplified by substitution of passive two-ports [62]. More complex nonlinear transfer curves can be implemented by digital subcircuits [63]. The method leading to full on-chip implementation of chaotic oscillator can be found in an example in paper [64]. Circuity realization of chaotic oscillator based on model (1) is shown by means of Figure 11(a). It contains two voltage-feedback operational amplifiers TL082, current feedback operational amplifier with the frequency compensation node (TZ) AD844, and two four quadrant analogue multipliers AD633 with versatile voltage transfer function $V_{W}=K \cdot\left(V_{X 1}-V_{X 2}\right)\left(V_{Y 1}-V_{Y 2}\right)$. All state variables can be easily measured as voltages at output of inverting integrators. Circuit is fed by symmetrical $\pm 15 \mathrm{~V}$ voltage supply. Dynamical behaviour of this network is described by the following set of ordinary differential equations:

$$
\begin{aligned}
& C \frac{\mathrm{d}}{\mathrm{d} t} v_{1}=-\frac{v_{1}}{R_{1}}-\frac{v_{2}}{R_{2}}, \\
& C \frac{\mathrm{d}}{\mathrm{d} t} v_{2}=-\frac{v_{1}}{R_{3}}-\frac{v_{3}}{R_{4}}+\frac{K^{2}}{R_{5}} v_{1}^{3}, \\
& C \frac{\mathrm{d}}{\mathrm{d} t} v_{3}=\frac{v_{2}}{R_{6}},
\end{aligned}
$$

where $K=0.1$ represents internally trimmed transfer constant of multiplier. 


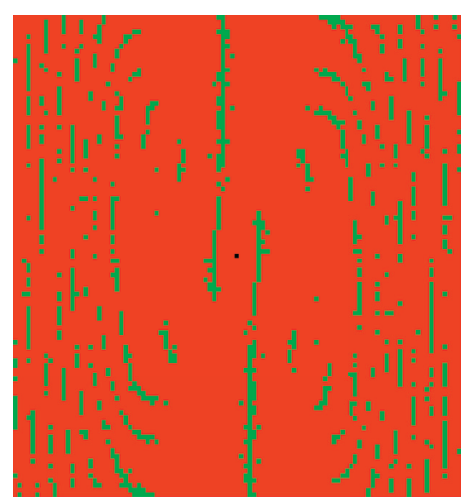

(a)

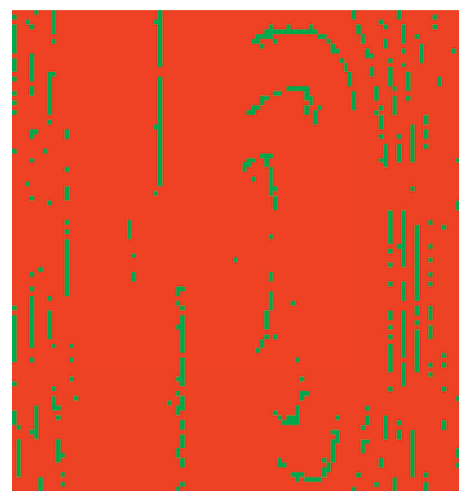

(d)

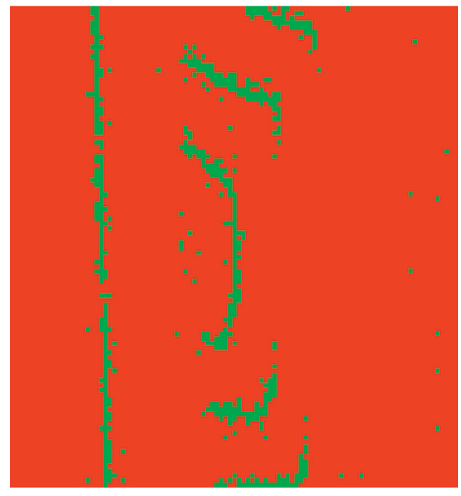

(g)

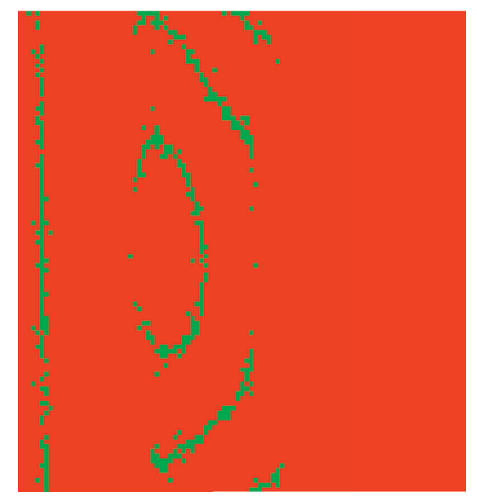

(j)

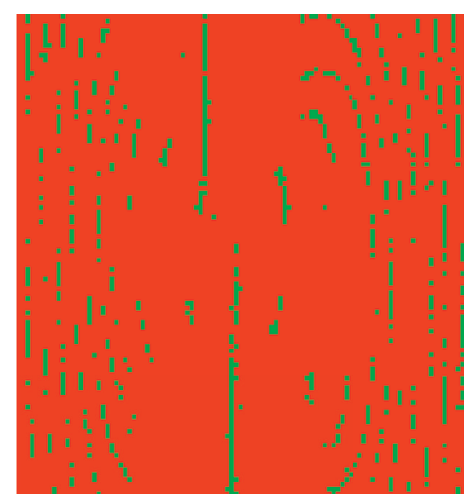

(b)

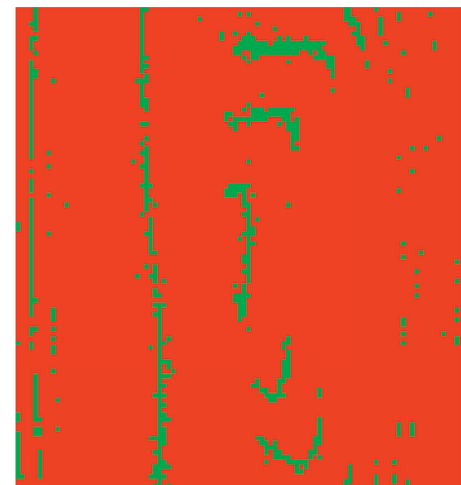

(e)

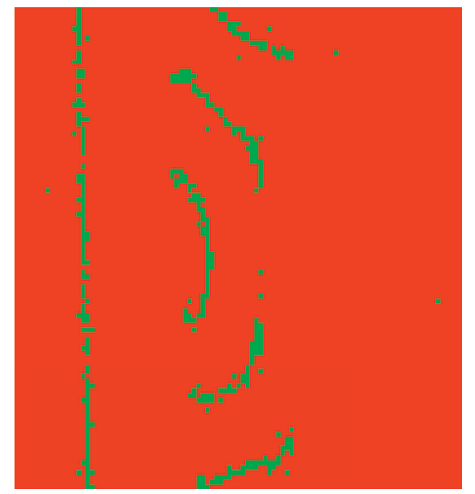

(h)

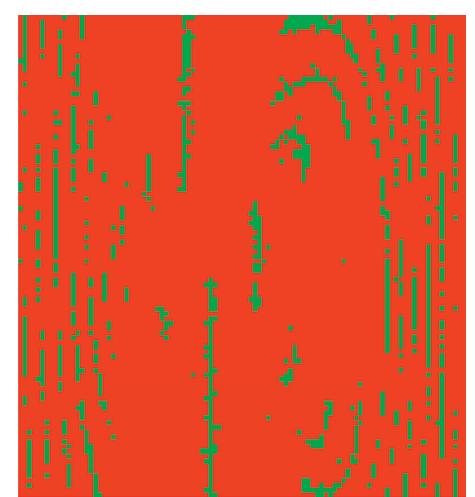

(c)

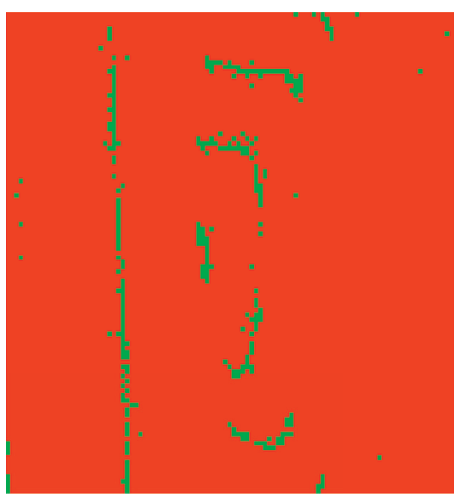

(f)

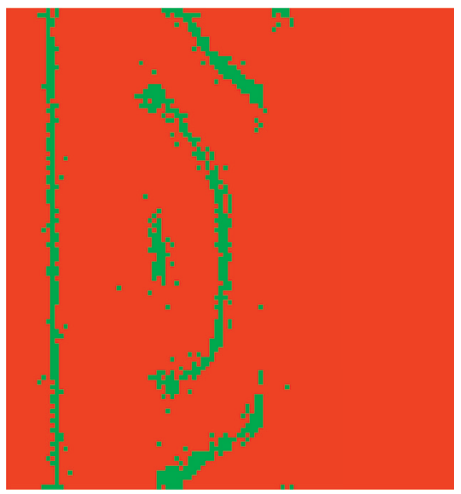

(i)

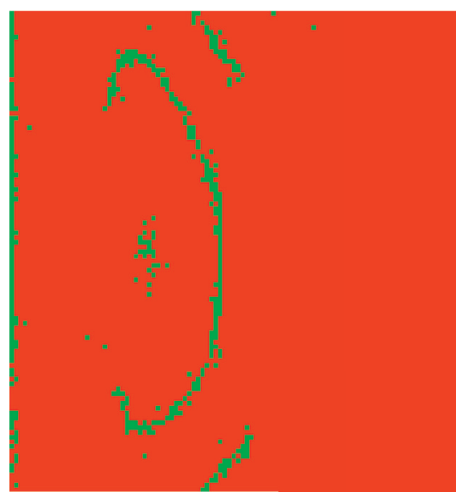

(k)

FIGURE 9: Basins of attraction visualized for list of horizontal planes: (a) $z_{0}=0$, (b) $z_{0}=0.5$, (c) $z_{0}=1$, (d) $z_{0}=1.5$, (e) $z_{0}=2$, (f) $z_{0}=2.5$, (g) $z_{0}=3$, (h) $z_{0}=3.5$, (i) $z_{0}=4$, (j) $z_{0}=4.5$, (k) $z_{0}=5$. 


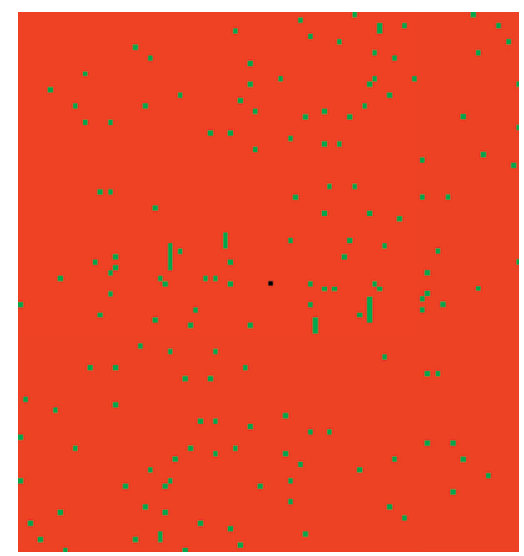

(a)

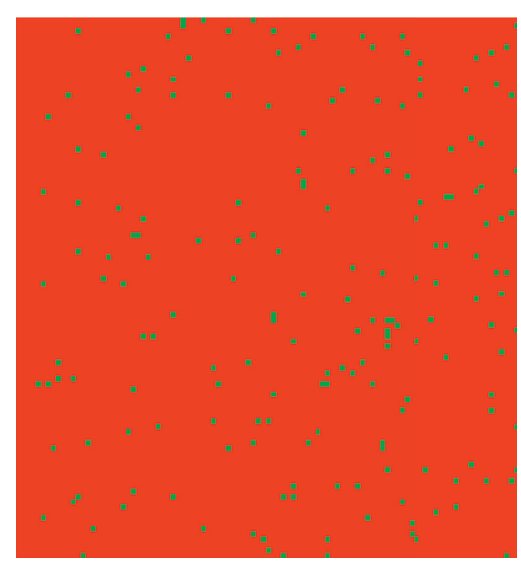

(d)

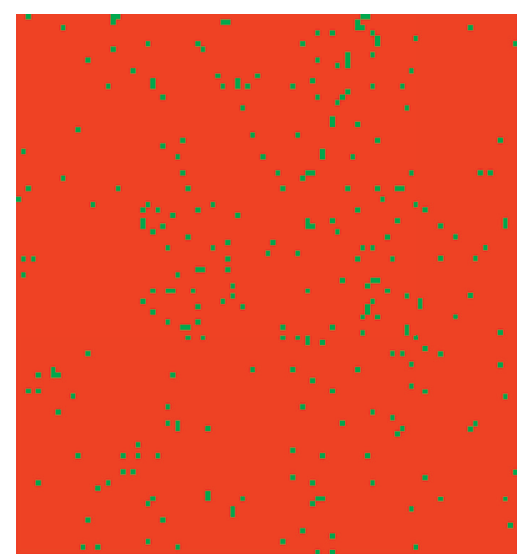

(b)

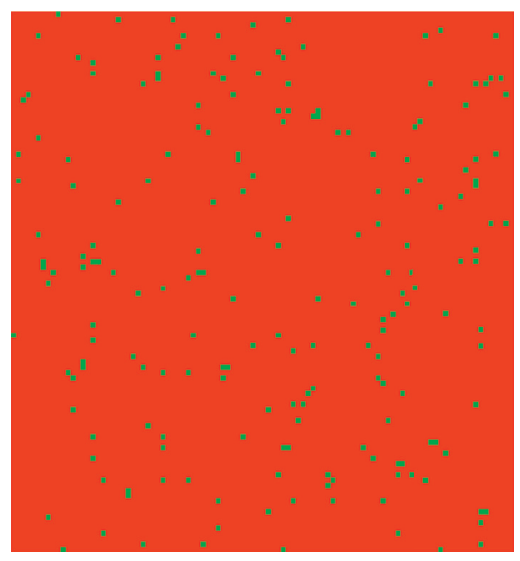

(e)

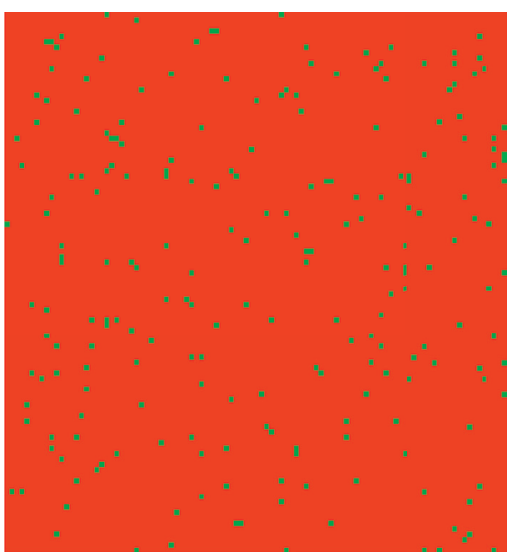

(c)

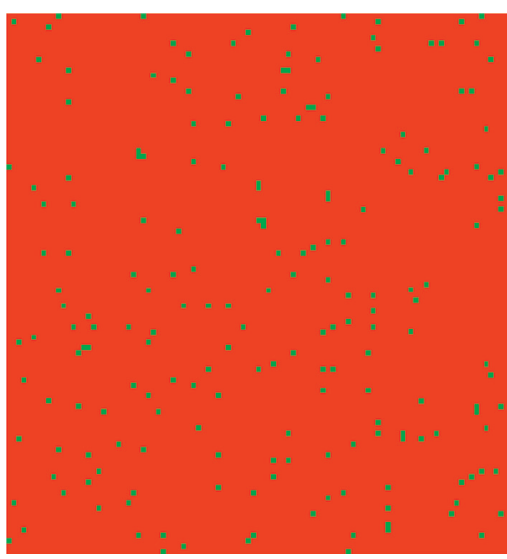

(f)

FIGURE 10: Basins of attraction visualized for list of horizontal planes: (a) $z_{0}=0$, (b) $z_{0}=1$, (c) $z_{0}=2$, (d) $z_{0}=3$, (e) $z_{0}=4$, (f) $z_{0}=5$.

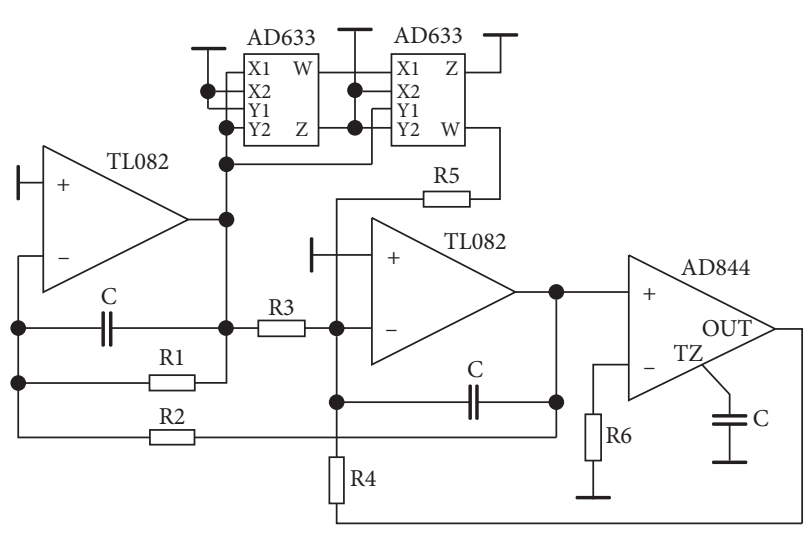

(a)

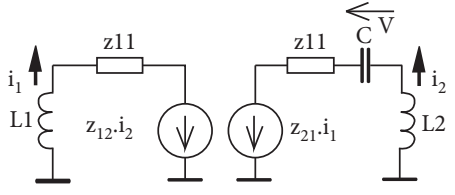

(b)

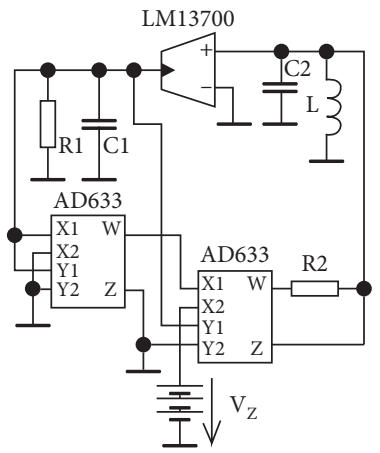

(c)

FIGURE 11: Two flow equivalent circuitry realizations of chaotic dynamical system: (a) voltage mode implementation of dynamical system (1), (b) principal schematic of dual circuit. 


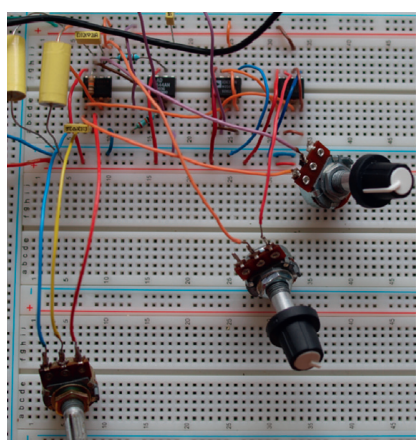

(a)

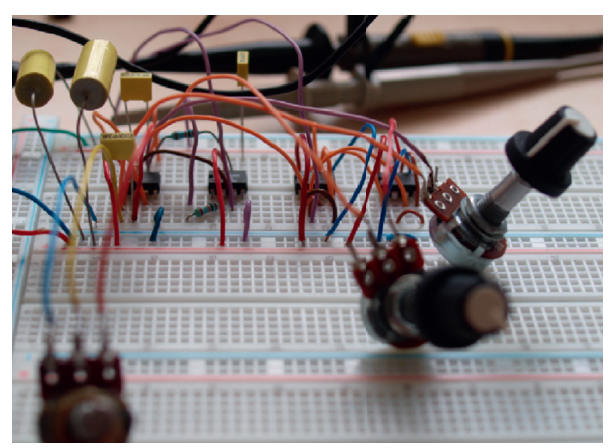

(b)

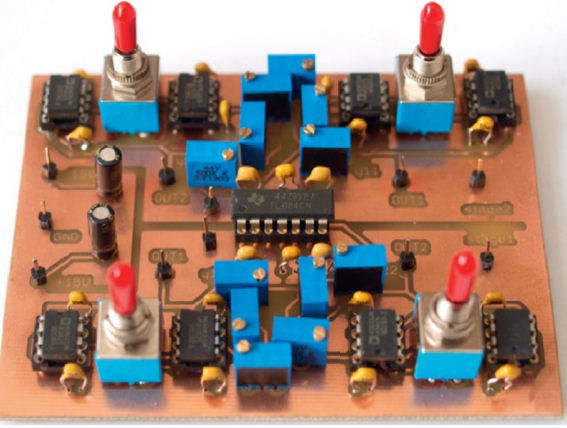

(c)

FiguRe 12: Experimental verification of discovered new chaotic oscillator: (a) (b) bread board circuit realization, (c) realization of single bipolar transistor-based class $\mathrm{C}$ amplifier cell with nonlinear transconductances.

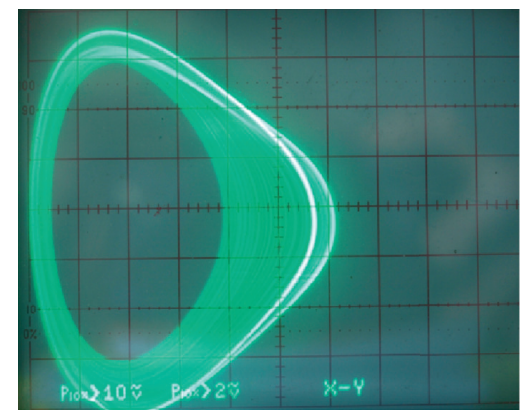

(a)

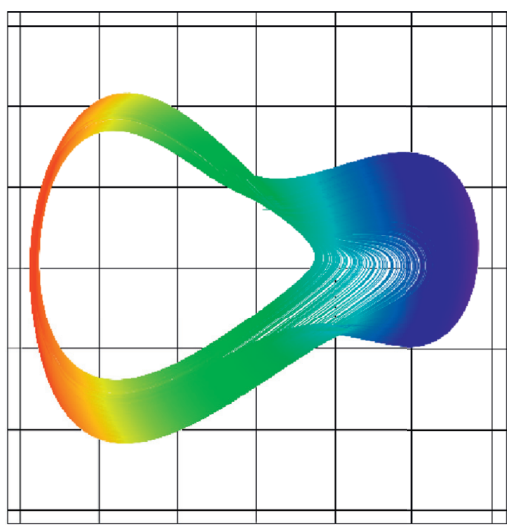

(d)

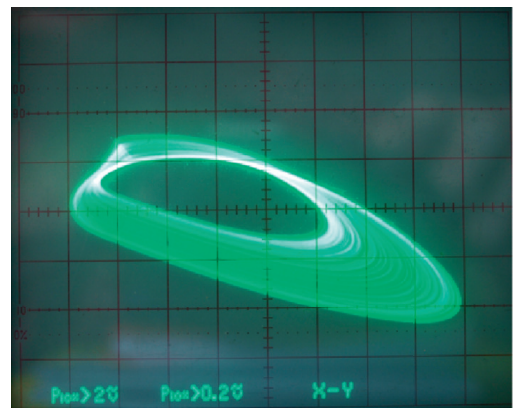

(b)

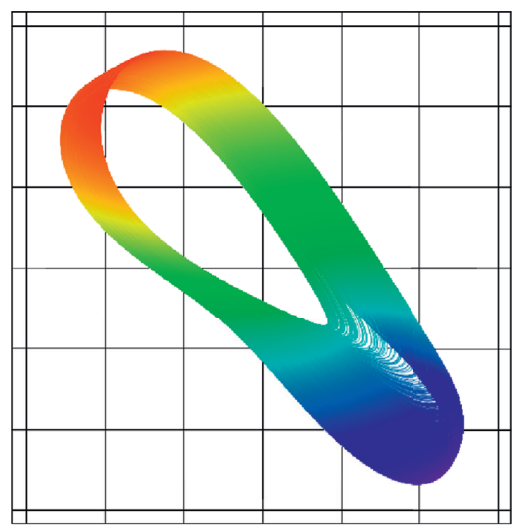

(e)

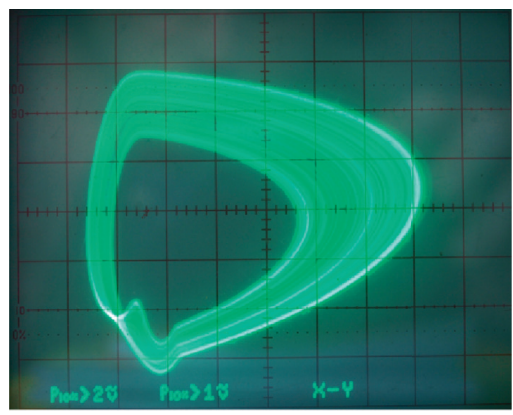

(c)

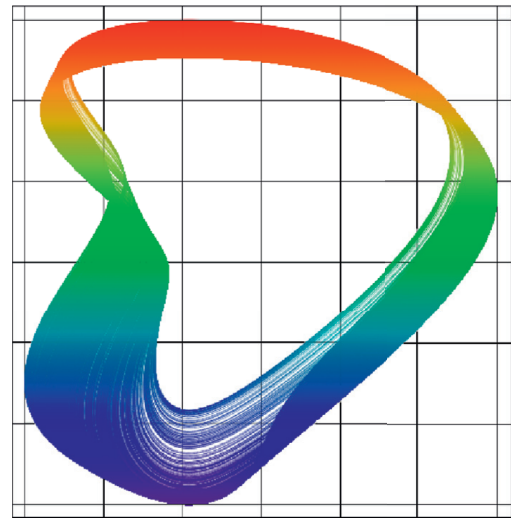

(f)

FIGURE 13: Experimental verification of designed circuit representation of class $\mathrm{C}$ amplifier, measured strange attractors in different plane projections: (a) $v_{2}$ vs $v_{1}$, (b) $v_{3}$ vs $v_{1}$, (c) $v_{3}$ vs $v_{2}$, and numerically integrated trajectories: (d) $v_{2}$ vs $v_{1}$ plane, (e) $v_{3}$ vs $v_{1}$ plane, (f) $v_{3}$ vs $v_{1}$ plotted with uniform voltage range $\pm 3 \mathrm{~V}$.

Nonideal and parasitic properties of used active devices clearly limit the frequency spectrum of generated signal. This could be especially important in the case of oscillators where output waveforms (state variables) are the broad-band noise-like chaotic signals. Fortunately, time constant of designed chaotic oscillator can be shortened and resulting waveforms judged, both using suitable circuit simulator or laboratory measurement. Because of better correspondence between theoretical expectations and circuit analysis, OrCAD PSpice program has been utilized for this purpose. Individual active elements were modelled using third level of abstraction and influential parasitic properties swept parametrically. From the viewpoint of designed circuit, the most problematic part is AD633 exhibiting heavily distorted input-output characteristics for time constant of chaotic oscillator shorter than $1 \mu \mathrm{s}$. Further interesting reading about this topic can be found in [65].

Figure 11(b) recalls duality principle and construct circuit that is dual to Figure 1(b). It comprises two currentcontrolled voltage sources, but only $z_{21}\left(i_{1}\right)$ is nonlinear. This circuit is described by the same differential equations as (1) 


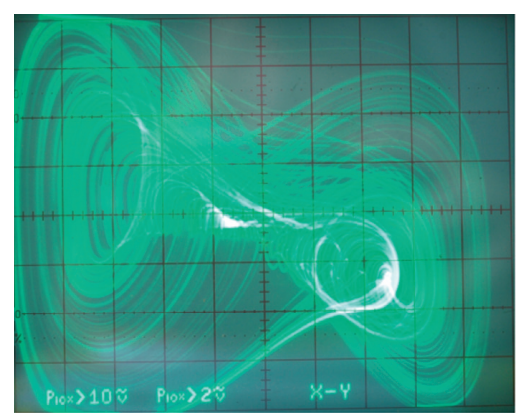

(a)

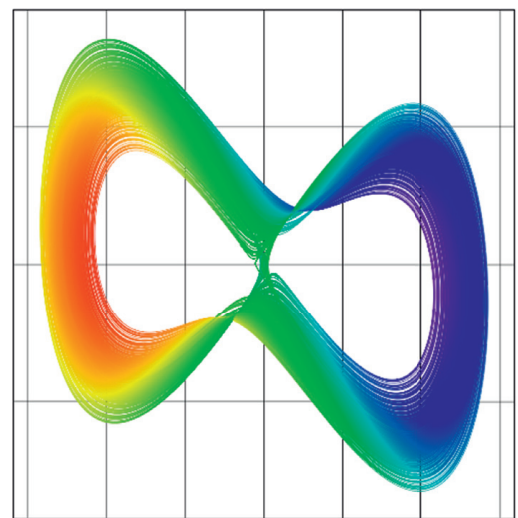

(d)

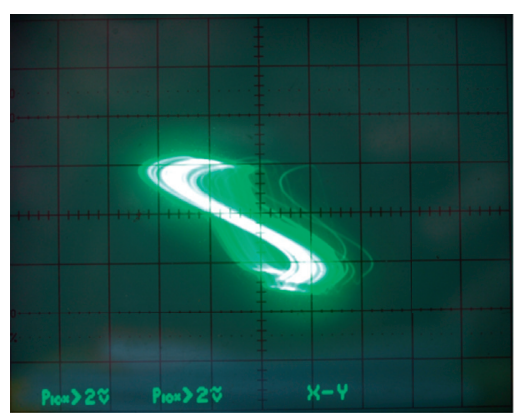

(b)

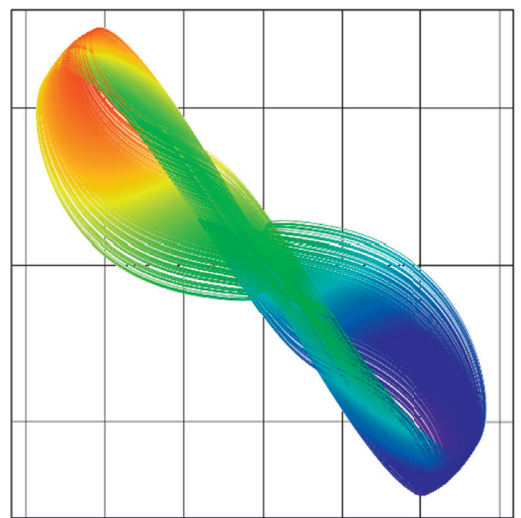

(e)

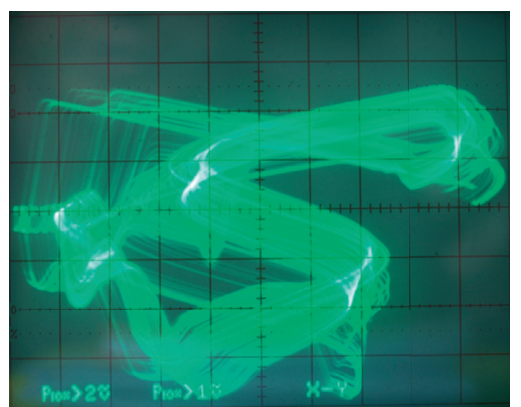

(c)

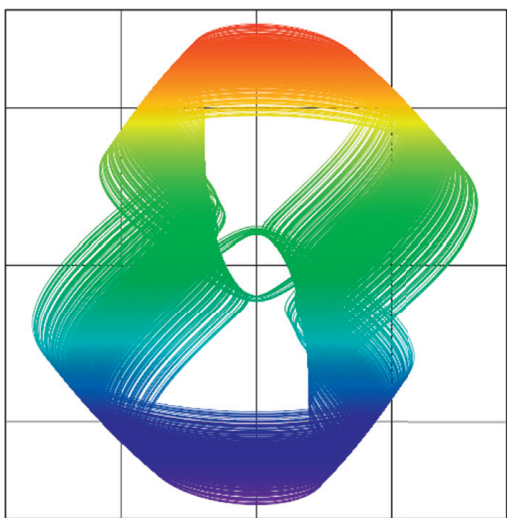

(f)

FIGURE 14: Experimental verification of designed circuit representation of class $\mathrm{C}$ amplifier, measured strange attractors in different plane projections: (a) $v_{2}$ vs $v_{1}$, (b) $v_{3}$ vs $v_{1}$, (c) $v_{3}$ vs $v_{2}$, and numerically integrated trajectories: (d) $v_{2}$ vs $v_{1}$ plane, (e) $v_{3}$ vs $v_{1}$ plane, (f) $v_{3}$ vs $v_{1}$ plotted with uniform voltage range $\pm 3 \mathrm{~V}$.

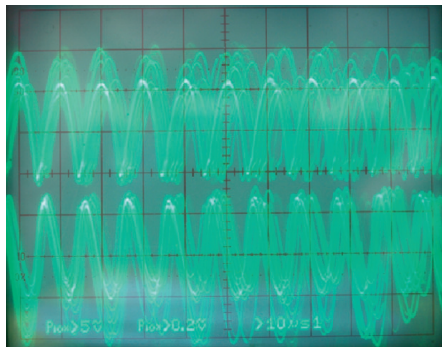

(a)

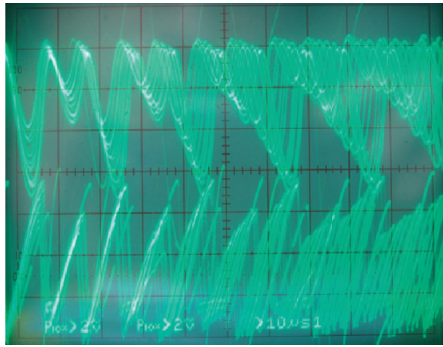

(b)

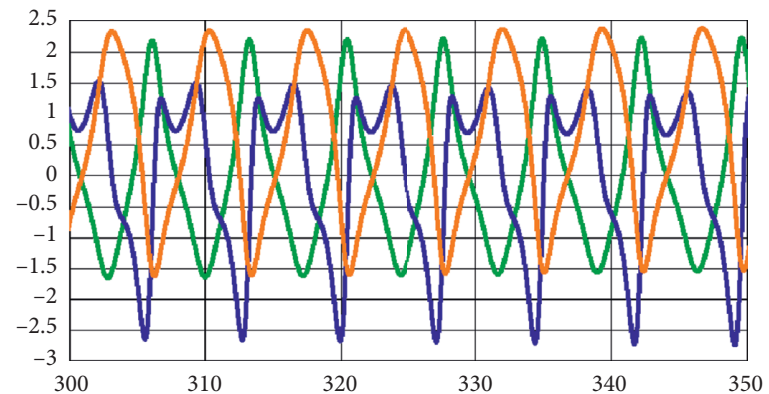

(c)

Figure 15: Chaotic waveforms in time domain captured by oscilloscope: (a) $v_{3}(t)$ and $v_{1}(t)$, (b) $v_{2}(t)$ and $v_{1}(t)$, numerically integrated orbits (c) $v_{1}(t)$ green, $v_{2}(t)$ blue, and $v_{3}(t)$ orange.

but after considering dual variables (voltages/currents) and parameters (admittances/impedances). In detail,

$$
\begin{aligned}
& L_{1} \frac{\mathrm{d}}{\mathrm{d} t} i_{1}=-z_{11} \cdot i_{1}-z_{12} \cdot i_{2}, \\
& L_{2} \frac{\mathrm{d}}{\mathrm{d} t} i_{2}=-z_{21} \cdot i_{1}-z_{22} \cdot i_{2}-v, \\
& C \frac{\mathrm{d}}{\mathrm{d} t} v=i_{2},
\end{aligned}
$$

where state vector becomes $x=\left(i_{1}, i_{2}, v\right)^{T}$. Direct circuitry realization of the state description of class $C$ amplifier (1) can be found in Figure 11(c). The corresponding set of the ordinary differential equations are

$$
\begin{aligned}
C_{1} \frac{\mathrm{d}}{\mathrm{d} t} v_{1} & =-\frac{v_{1}}{R_{1}}-g_{T} \cdot v_{2}, \\
C_{2} \frac{\mathrm{d}}{\mathrm{d} t} v_{2} & =\frac{1}{R_{2}}\left(K^{2} \cdot v_{1}^{3}-K \cdot V_{Z} \cdot v_{1}\right)-i_{L}, \\
L \frac{\mathrm{d}}{\mathrm{d} t} i_{L} & =v_{2},
\end{aligned}
$$




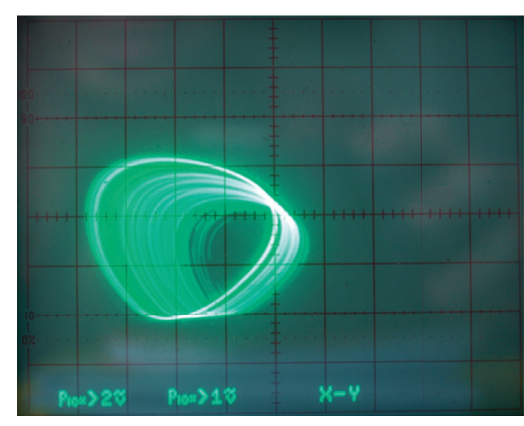

(a)

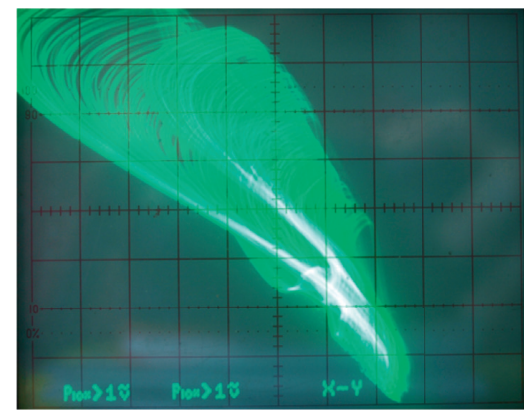

(d)

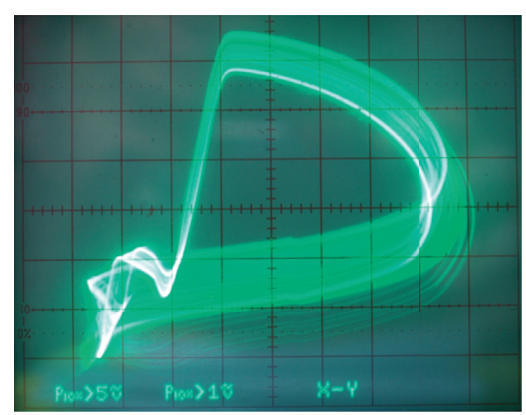

(g)

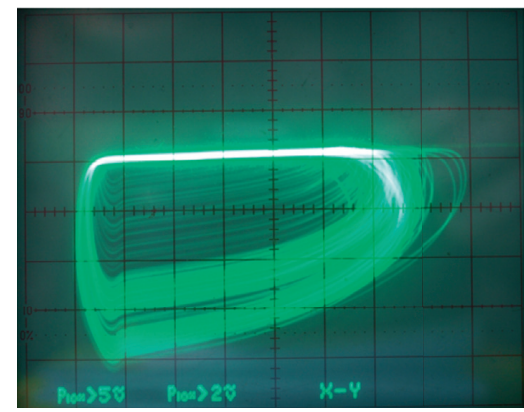

(j)

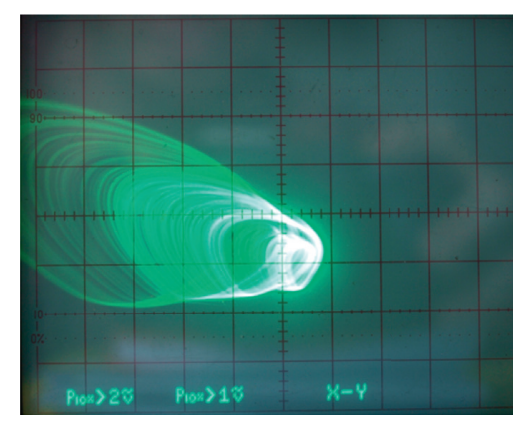

(b)

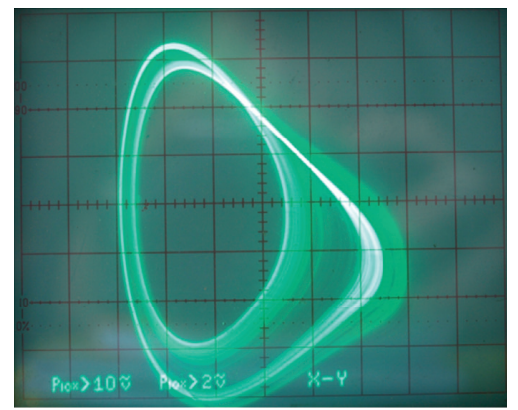

(e)

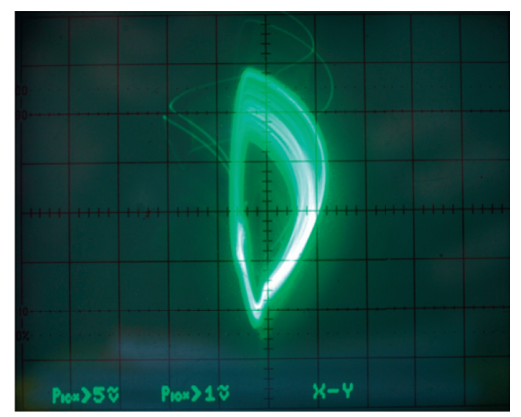

(h)

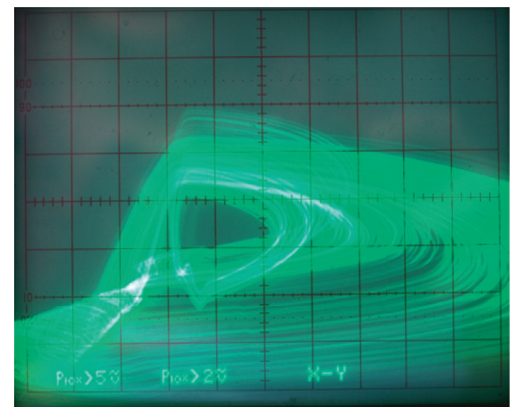

(k)

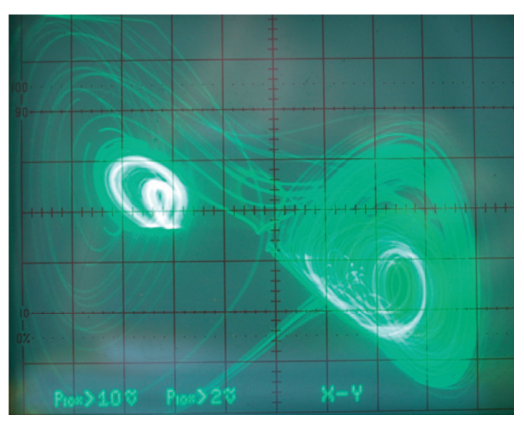

(c)

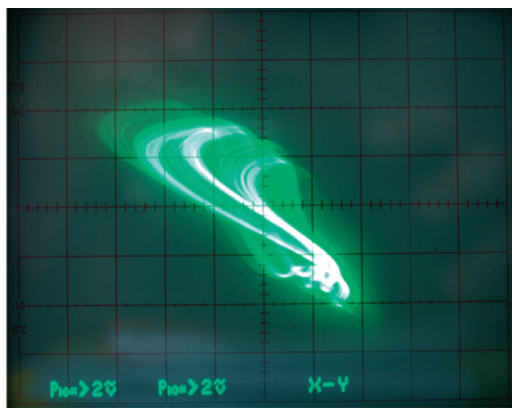

(f)

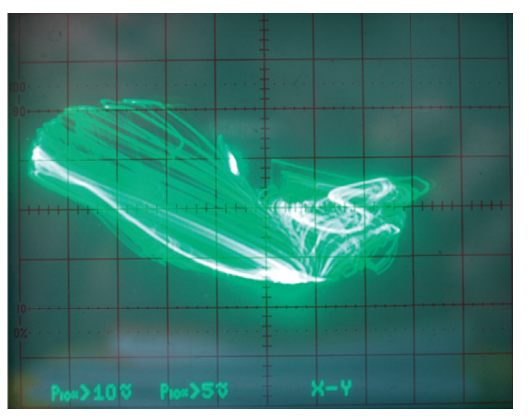

(i)

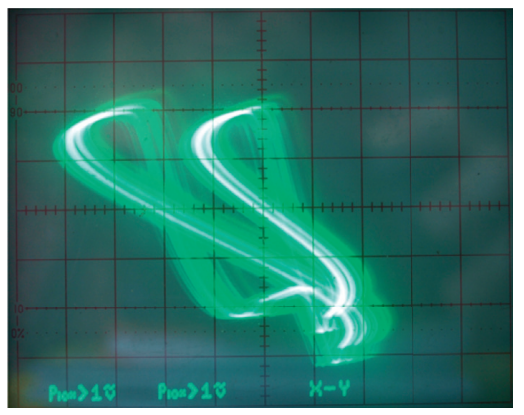

(l)

FIGURE 16: Geometrically interesting, strange attractors observed during experimental measurement but unnoticed in the frame of numerical analysis, different plane projections (not specified).

where $g_{T}$ is transconductance of operational transconductance amplifier LM13700 precisely adjusted by external DC current. Because the desired strange attractors are located inside a cube with edges $\pm 3 \mathrm{~V}$, dynamical range of integrated circuit LM13700 does not present a problem. The whole chaotic circuit can be supplied by symmetrical $\pm 15 \mathrm{~V}$. To turn around output current of LM13700, input voltage nodes should be swapped.

\section{Experimental Verification}

The chaotic oscillator proposed in the previous section was constructed using breadboard; see Figure 12. For experimental verification, time constant was set to $\tau=10 \mu \mathrm{s}$. This fact leads to choosing all capacitors $10 \mathrm{nF}$ and, using basic calculations, values of remaining passive circuit components: $R_{1}=50 \mathrm{k} \Omega, R_{2}=10 \mathrm{k} \Omega, \quad R_{3}=3.9 \mathrm{k} \Omega, \quad R_{4}=10 \mathrm{k} \Omega$, 
$R_{5}=127 \Omega$, and $R_{6}=10 \mathrm{k} \Omega$. To trace evolution of chaos, two resistors are considered variable, namely, $R_{3}$ (parameter $b$ ) and $R_{5}$ (parameter $a$ ). Figure 12 also shows realization of printed circuit board of two-port described by admittance parameters with fully adjustable transconductances; i.e., shapes of both $y_{12}\left(v_{1}\right)$ and $y_{21}\left(v_{1}\right)$ can be adjusted independently. Individual measurement results are provided by means of Figures 13-16.

\section{Conclusion}

Starting with a very simple mathematical model of class C amplifier with single bipolar transistor, two geometrically distinct strange attractors have been discovered. There are two conditions for chaos evolution: non-unilaterality and nonlinear forward transconductance of transistor. Numerical analysis includes calculation of LLE, energy distributions, and basins of attraction.

Based on this finding, a robust chaos generator is designed and experimentally verified within this paper. Circuitry realization follows analogue computer concept and utilizes cheap and commercially available active elements. Several different shapes of the self-excited strange attractors were observed during experimental verification.

The problem solved in this work opens new topics for future research. For example, existence of chaos in common building blocks dedicated for analogue signal processing such as multi-stage amplifiers, cascodes, current mirrors, and differential amplifiers could be proven via the same fully numerical approach as proposed in this paper. Also, staying focused on topology of class C amplifier, "chaotic" configurations of internal parameters that are closely related to standard operational regime could be localized. Particular attention should be paid to the promising cases where backward transconductance is very low. Finally, input signal as a driving force acts as an additional degree of freedom that can increase the overall probability of chaotic solution.

\section{Data Availability}

There are two groups of results (data outputs) presented in this manuscript. 1. Those coming from numerical routines used for mathematical analysis of investigated dynamical system (integration of state trajectories, sensitivity properties, calculation of the largest Lyapunov exponent, and visualization of basins of attraction). 2. Experimental verification of designed chaotic oscillator. This type of output can be resimulated by using OrCAD PSpice circuit simulator. In both cases, source files, schematics, and simulation profiles can be forwarded to candidates based on the e-mail request submitted to the author of this paper, that is, petrzelj@feec.vutbr. cz. Please do not hesitate to contact the author also in the case of some questions, suggestions, and comments. Details about input parameters for numerical procedures can be found in the main body of the paper.

\section{Conflicts of Interest}

The author declares that there are no conflicts of interest regarding the publication of this paper.

\section{Acknowledgments}

The author would like to thank Dr. Tomas Gotthans for his long-time support, much useful advice, and recommendations about the concept of this paper. The research described in this paper was financed by Grant Agency of Czech Republic under project no. 19-22248S. For research, the infrastructure of the SIX Center was used.

\section{References}

[1] M. P. Kennedy, "Chaos in the colpitts oscillator," IEEE Transactions on Circuits and Systems I: Fundamental Theory and Applications, vol. 41, no. 11, pp. 771-774, 1994.

[2] P. Kvarda, "Chaos in hartley's oscillator," International Journal of Bifurcation and Chaos, vol. 12, no. 10, pp. 22292232, 2002.

[3] R. Kiliç and F. Yildirim, "A survey of wien bridge-based chaotic oscillators: design and experimental issues," Chaos, Solitons \& Fractals, vol. 38, no. 5, pp. 1394-1410, 2008.

[4] Ö. Morgül, "Wien bridge based RC chaos generator," Electronics Letters, vol. 31, no. 24, pp. 2058-2059, 1995.

[5] G.-Q. Zhong, "Implementation of chua's circuit with a cubic nonlinearity," IEEE Transactions on Circuit and Systems I: Fundamental Theory and Applications, vol. 41, no. 12, pp. 934-941, 1994.

[6] L. O. Chua and G.-N. Lin, "Canonical realization of chua's circuit family," IEEE Transactions on Circuits and Systems, vol. 37, no. 7, pp. 885-902, 1990.

[7] A. S. Elwakil and M. P. Kennedy, "Chaotic oscillator configuration using a frequency dependent negative resistor," Journal of Circuits, Systems and Computers, vol. 9, pp. 229242, 1999.

[8] J. Petrzela, Z. Kolka, and S. Hanus, "Simple chaotic oscillator: from mathematical model to practical experiment," Radioengineering, vol. 15, no. 1, pp. 6-12, 2006.

[9] A. S. Elwakil and M. P. Kennedy, "A semi-systematic procedure for producing chaos from sinusoidal oscillators using diode-inductor and FET-capacitor composites," IEEE Transactions on Circuits and Systems I: Fundamental Theory and Applications, vol. 47, no. 4, pp. 582-590, 2000.

[10] A. S. Elwakil and M. P. Kennedy, "Novel chaotic oscillator configuration using a diode-inductor composite," International Journal of Electronics, vol. 87, no. 4, pp. 397-406, 2010.

[11] P. Bernat and I. Balaz, "RC autonomous circuits with chaotic behavior," Radioengineering, vol. 11, no. 2, pp. 1-5, 2002.

[12] A. S. Elwakil and M. P. Kennedy, "Chaotic oscillators derived from sinusoidal oscillators based on the current feedback op amp," Analog Integrated Circuits and Signal Processing, vol. 24, no. 3, pp. 239-251, 2000.

[13] E. Fossas and G. Olivar, "Study of chaos in the buck converter," IEEE Transactions on Circuits and Systems I: Fundamental Theory and Applications, vol. 43, no. 1, pp. 13-25, 1996.

[14] C. K. Tse and W. C. Y. Chan, "Chaos from a current-programmed ćuk converter," International Journal of Circuit Theory and Applications, vol. 23, no. 3, pp. 217-225, 1995.

[15] J. H. B. Deane, "Chaos in a current-mode controlled boost DC-DC converter," IEEE Transactions on Circuits and Systems I: Fundamental Theory and Applications, vol. 39, no. 8, pp. 680-683, 1992.

[16] C. K. Tse, "Flip bifurcation and chaos in three-state boost switching regulators," IEEE Transactions on Circuits and 
Systems I: Fundamental Theory and Applications, vol. 41, no. 1, pp. 16-23, 1994.

[17] X. Zhou, J. Li, and Y. M, "Chaos phenomena in DC-DC converter and chaos control," Procedia Engineering, vol. 29, no. 12 , pp. $470-473,2012$.

[18] M. Di Bernardo, F. Garefalo, L. Glielmo, and F. Vasca, "Switchings, bifurcations, and chaos in DC/DC converters," IEEE Transactions on Circuits and Systems I: Fundamental Theory and Applications, vol. 45, no. 2, pp. 133-141, 1998.

[19] D. C. Hamill and D. J. Jeffries, "Subharmonics and chaos in a controlled switched-mode power converter," IEEE Transactions on Circuits and Systems, vol. 35, no. 8, pp. 1059-1061, 1988.

[20] A. Rodriguez-Vazquez, J. Huertas, and L. Chua, "Chaos in switched-capacitor circuit," IEEE Transactions on Circuits and Systems, vol. 32, no. 10, pp. 1083-1085, 1985.

[21] M. Drutarovsky and P. Galajda, "A robust chaos-based true random number generator embedded in reconfigurable switched-capacitor hardware," Radioengineering, vol. 16, no. 3, pp. 120-127, 2007.

[22] T. Endo and L. O. Chua, "Chaos from phase-locked loops," IEEE Transactions on Circuits and Systems, vol. 35, no. 8, pp. 987-1003, 1988.

[23] T. Endo, "A review of chaos and nonlinear dynamics in phaselocked loops," Journal of the Franklin Institute, vol. 331, no. 6, pp. 859-902, 1994.

[24] J. Petrzela, "Multi-valued static memory with resonant tunneling diodes as natural source of chaos," Nonlinear Dynamics, vol. 94, no. 3, pp. 1867-1887, 2018.

[25] J. Petrzela, "Strange attractors generated by multiple-valued static memory cell with polynomial approximation of resonant tunneling diodes," Entropy, vol. 20, no. 9, pp. 697-719, 2018.

[26] P. Galajda, M. Guzan, and V. Spany, "The state space mystery with negative load in multiple-valued logic," Radioengineering, vol. 8, no. 2, pp. 2-7, 1999.

[27] S. C. Yener and H. H. Kuntman, "Fully CMOS memristor based chaotic circuit," Radioengineering, vol. 23, no. 4, pp. 1140-1149, 2014.

[28] B. Muthuswamy and L. O. Chua, "Simplest chaotic circuit," International Journal of Bifurcation and Chaos, vol. 20, no. 5, pp. 1567-1580, 2010.

[29] Z. Biolek, D. Biolek, and V. Biolkova, "Differential equations of ideal memristors," Radioengineering, vol. 24, no. 2, pp. 369-377, 2015.

[30] Z. Biolek and D. Biolek, "Euler-lagrange equations of networks with higher-order elements," Radioengineering, vol. 26, no. 2, pp. 397-405, 2017.

[31] K. Rajagopal, C. Li, F. Nazarimehr, A. Karthikeyan, P. Duraisamy, and S. Jafari, "Chaotic dynamics of modified wien bridge oscillator with fractional order memristor," Radioengineering, vol. 27, no. 1, pp. 165-174, 2019.

[32] J. Petrzela, "On the existence of chaos in the electronically adjustable structures of the state variable filters," International Journal of Circuit Theory and Applications, vol. 44, no. 10, pp. 1779-1797, 2016.

[33] M. J. Ogorzalek, "Order and chaos in a third-order RC ladder network with nonlinear feedback," IEEE Transactions on Circuits and Systems, vol. 36, no. 9, pp. 1221-1230, 1989.

[34] G.-Q. Zhong, K.-T. Ko, K.-F. Man, and K.-S. Tang, “A systematic procedure for synthesizing two-terminal devices with polynomial non-linearity," International Journal of Circuit Theory and Applications, vol. 29, no. 2, pp. 241-249, 2001.
[35] J. Petrzela and L. Polak, "Minimal realizations of autonomous chaotic oscillators based on trans-immittance filters," IEEE Access, vol. 7, no. 1, pp. 17561-17577, 2019.

[36] T. Matsumoto, "Chaos in electronic circuits," Proceedings of the IEEE, vol. 75, no. 8, pp. 1033-1057, 1987.

[37] H. P. W. Gottlieb, "What is the simplest jerk function that gives chaos?" American Journal of Physics, vol. 64, no. 5, p. 525, 1996.

[38] L. Minati, M. Frasca, P. Oświ $\square$ cimka, L. Faes, and S. Drożdż, "Atypical transistor-based chaotic oscillators: design, realization, and diversity," Chaos: An Interdisciplinary Journal of Nonlinear Science, vol. 27, no. 7, Article ID 73113, 2017.

[39] L. Keuninckx, G. Van der Sande, and J. Danckaert, "Simple two-transistor single-supply resistor-capacitor chaotic oscillator," IEEE Transactions on Circuits and Systems II: Express Briefs, vol. 62, no. 9, pp. 891-895, 2015.

[40] J. Petrzela, "Generalized single stage class c amplifier: analysis from the viewpoint of chaotic behavior," Applied Sciences, vol. 10, no. 15, p. 5025, 2020.

[41] K. Grygiel and P. Szlachetka, "Lyapunov exponents analysis of autonomous and nonautonomous sets of ordinary differential equations," Acta Physica Polonica Series B, vol. 26, no. 8, pp. 1321-1331, 1995.

[42] S. Jafari, J. C. Sprott, V.-T. Pham, and S. M. R. H. Golpayegani, "A new cost function for parameter estimation of chaotic systems using return maps as fingerprints," International Journal of Bifurcation and Chaos, vol. 24, no. 10, pp. 15671580, 2014.

[43] S. Panahi, S. Jafari, V.-T. Pham, S. T. Kingni, A. Zahedi, and S. H. Sedighy, "Parameter identification of a chaotic circuit with a hidden attractor using krill herd optimization," International Journal of Bifurcation and Chaos, vol. 26, no. 13, Article ID 1650221, 2016.

[44] P. Bartissol and L. O. Chua, "The double hook (nonlinear chaotic circuits)," IEEE Transactions on Circuits and Systems, vol. 35, no. 12, pp. 1512-1522, 1988.

[45] I. Podlubny, "Matrix approach to discrete fractional calculus," Fractional Calculus and Applied Analysis, vol. 3, no. 4, pp. 359-386, 2000.

[46] I. Podlubny, A. Chechkin, T. Skovranek, Y. Chen, and B. M. Vinagre Jara, "Matrix approach to discrete fractional calculus II: partial fractional differential equations," Journal of Computational Physics, vol. 228, no. 8, pp. 3137-3153, 2009.

[47] H. Lu, J. Petrzela, T. Gotthans, K. Rajagopal, S. Jafari, and I. Hussain, "Fracmemristor chaotic oscillator with multistable and antimonotonicity properties," Journal of Advanced Research, vol. 25, pp. 137-145, 2020.

[48] J. M. Muñoz-Pacheco, "Infinitely many hidden attractors in a new fractional-order chaotic system based on a fracmemristor," The European Physical Journal Special Topics, vol. 228, no. 10, pp. 2185-2196, 2019.

[49] J. Munoz-Pacheco, E. Zambrano-Serrano, C. Volos, S. Jafari, J. Kengne, and K. Rajagopal, "A new fractional-order chaotic system with different families of hidden and self-excited attractors," Entropy, vol. 20, no. 8, p. 564, 2018.

[50] C. Volos, V.-T. Pham, E. Zambrano-Serrano, J. M. MunozPacheco, S. Vaidyanathan, and E. Tlelo-Cuautle, "Analysis of a 4-D hyperchaotic fractional-order memristive system with hidden attractors," Advances in Memristors, Memristive Devices and Systems, vol. 701, pp. 207-235, 2017.

[51] J. Mou, K. Sun, H. Wang, and J. Ruan, "Characteristic analysis of fractional-order 4D hyperchaotic memristive circuit," Mathematical Problems in Engineering, vol. 2017, Article ID 2313768, 13 pages, 2017. 
[52] A. Kartci, N. Herencsar, J. T. Machado, and L. Brancik, "History and progress of fractional-order element passive emulators: a review," Radioengineering, vol. 29, no. 2, pp. 296-304, 2020.

[53] I. Podlubny, I. Petráś, B. M. Vinagre, P. O’Leary, and L. Dorčák, "Analogue realizations of fractional-order controllers," Nonlinear Dynamics, vol. 29, no. 1, pp. 281-296, 2002.

[54] J. Petrzela, "Fractional-order chaotic memory with wideband constant phase elements," Entropy, vol. 22, no. 4, p. 422, 2020.

[55] J. Petrzela, "Optimal piecewise-linear approximation of quadratic chaotic dynamics," Radioengineering, vol. 21, no. 1, pp. 20-28, 2012.

[56] J. Lü and G. Chen, "Generating multiscroll chaotic attractors: theories, methods and applications," International Journal of Bifurcation and Chaos, vol. 16, no. 04, pp. 775-858, 2006.

[57] W. Deng and J. Lü, "Generating multi-directional multi-scroll chaotic attractors via a fractional differential hysteresis system," Physics Letters A, vol. 369, no. 5, pp. 438-443, 2007.

[58] J. C. Sprott, "A proposed standard for the publication of new chaotic systems," International Journal of Bifurcation and Chaos, vol. 21, no. 9, pp. 2391-2394, 2011.

[59] J. Petrzela, Z. Hrubos, and T. Gotthans, "Modeling deterministic chaos using electronic circuits," Radioengineering, vol. 20, no. 2, pp. 438-444, 2011.

[60] M. Itoh, "Synthesis of electronic circuits for simulating nonlinear dynamics," International Journal of Bifurcation and Chaos, vol. 11, no. 3, pp. 605-653, 2001.

[61] J. Petrzela, T. Gotthans, and M. Guzan, "Current-mode network structures dedicated for simulation of dynamical systems with plane continuum of equilibrium," Journal of Circuits, Systems and Computers, vol. 27, no. 9, Article ID 1830004, 2018.

[62] J. C. Sprott, “A new class of chaotic circuit,” Physics Letters A, vol. 266, no. 1, pp. 19-23, 2000.

[63] T. Gotthans and J. Petrzela, "Experimental study of the sampled labyrinth chaos," Radioengineering, vol. 20, no. 4, pp. 873-879, 2011.

[64] R. Trejo-Guerra, E. Tlelo-Cuautle, V. H. Carbajal-Gómez, and G. Rodriguez-Gómez, "A survey on the integrated design of chaotic oscillators," Applied Mathematics and Computation, vol. 219, no. 10, pp. 5113-5122, 2013.

[65] J. M. Muñoz-Pacheco, E. Tlelo-Cuautle, I. Toxqui-Toxqui, C. Sánchez-López, and R. Trejo-Guerra, "Frequency limitations in generating multi-scroll chaotic attractors using CFOAs," International Journal of Electronics, vol. 101, no. 11, pp. 1559-1569, 2014. 